\title{
New perspectives on the supernova remnant G78.2+2.1
}

\author{
Y. Ladouceur and S. Pineault
}

\begin{abstract}
Département de physique, de génie physique et d'optique et Centre de recherche en astrophysique du Québec (CRAQ), Université Laval, Québec (Qué), G1V 0A6, Canada e-mail: pineault@phy.ulaval.ca; yvan.ladouceur@dgpc.ulaval.ca
\end{abstract}

Received 14 July 2008 / Accepted 15 August 2008

\section{ABSTRACT}

\begin{abstract}
Aims. The Galactic object G78.2+2.1 is a classical shell-type supernova remnant. Previous radio continuum studies have shown substantial variations in spectral index across the object. Existing studies of the neutral hydrogen are of relatively low resolution and sensitivity. Much higher sensitivity and resolution data from the Canadian Galactic Plane Survey justify a re-examination of the properties of this SNR.

Methods. In order to improve the accuracy of spectral index determination and its spatial variation, we first remove the underlying thermal emission with the use of a correlation between the $60 \mu \mathrm{m}$ infrared continuum and $1420 \mathrm{MHz}$ radio continuum. We use the so-called TT-plot technique to obtain the continuum spectral index between 408 and $1420 \mathrm{MHz}$. We also apply a "tomography" technique to investigate the spectral index variations, while keeping the full resolution of the data. Complementary X-ray and infrared data are used to study associated structures in both continuum and in the $21 \mathrm{~cm}$ line of neutral hydrogen.

Results. The spectral index $\alpha\left(S_{v} \propto v^{-\alpha}\right)$ averaged over the SNR is $0.75 \pm 0.03$, significantly higher than previous studies. The TT-plots and tomography image show spatial variations ranging from as low as 0.40 to as high as 0.80 . Two complementary neutral hydrogen structures at LSR velocities of 5.5 and $-14 \mathrm{~km} \mathrm{~s}^{-1}$, respectively, are shown to circumscribe the continuum emission. A number of scenarios are presented to account for the observed properties. A faint shell to the north-west of the SNR is visible in both radio and infrared continuum as well as in neutral hydrogen, and could be associated with a local blowout of the SNR blast wave into a medium of lower density. An isolated cloudlet probably accelerated by the SNR blast wave shows considerable structure in neutral hydrogen emission, suggesting the cloudlet is either beginning to dissolve within the SNR or is interacting with neighbouring gas. The previously proposed cloudlet at $\sim-117 \mathrm{~km} \mathrm{~s}^{-1}$ is not isolated, but is part of a more extended structure, and is thus unlikely an accelerated cloudlet.
\end{abstract}

Key words. ISM: supernova remnants - radio continuum: ISM - radio lines: ISM - ISM: bubbles - ISM: clouds

\section{Introduction}

The radio source G78.2+2.1 is one of the best examples of a shell-type supernova remnant (SNR). In one of the first radio studies of the Cygnus $\mathrm{X}$ region undertaken with the NRAO 140-ft telescope at $2695 \mathrm{MHz}$ and an angular resolution of 11', Wendker (1970) reported the presence of nonthermal emission in this direction (G78.2+1.8 and G78.4+2.5). Using $10.6 \mathrm{GHz}$ observations obtained with the Algonquin Radio Telescope, Higgs (1977a) confirmed the non-thermal nature of these two sources and further suggested they might be the brighter parts of a single extended SNR. This was confirmed by Higgs et al. (1977b) who used the DRAO Synthesis Telescope (Roger et al. 1973) to image the SNR at $1420 \mathrm{MHz}$ with arcmin resolution and showed it to have a nearly circular outline of about $60^{\prime}$ in diameter. In addition, using a global technique of separation into thermal and non-thermal components to estimate the spectral index between 1.4 and $10.6 \mathrm{GHz}$, Higgs et al. (1977b) found the best fit obtained for $\beta_{\mathrm{t}}=2.05$ and $\beta_{\mathrm{nt}}=2.65$, where $\beta$ is the brightness temperature spectral index $\left(T_{v} \propto v^{-\beta}\right)$, and subscripts $\mathrm{t}$ and $\mathrm{nt}$ refer to the thermal and non-thermal components, respectively. Note however that this technique is global in nature, in that it yields average values of the thermal and nonthermal components that best fit the SNR over its entire extent. The index $\beta$ is related to the usual flux density spectral index $\alpha$ by the relation $\beta=\alpha+2$, where we take $S_{v} \propto v^{-\alpha}$.
Using the DRAO Synthesis Telescope, Chastenay \& Pineault (1988), Pineault \& Chastenay (1990) and Wendker et al. (1991) obtained images of the remnant at $408 \mathrm{MHz}$. A comparison with the 1420-MHz image of Higgs et al. (1977b) shows a striking similarity between the two images. From these images, Pineault \& Chastenay (1990) derived a spectral index $\alpha$ of $0.46 \pm 0.16$, which should be considered as a lower limit since their $408 \mathrm{MHz}$ image did not contain short-spacing data. In their more detailed study, Wendker et al. (1991) found an integrated spectral index of $\alpha=0.52 \pm 0.06$ with no evidence of spatial variations exceeding \pm 0.1 . One of the most thorough studies of the radio continuum spectral index of the SNR was carried out by Zhang et al. (1997) using images obtained at four different frequencies from $232 \mathrm{MHz}$ to $4.8 \mathrm{GHz}$. They found an integrated spectral index of $\alpha=0.54 \pm 0.02$ and systematic spatial variations of the order of \pm 0.15 . Kothes et al. (2006) published a catalogue of Galactic SNRs present in the Canadian Galactic Plane Survey (CGPS - Taylor et al. 2003) in which they obtain $\alpha=0.64 \pm$ 0.09 based on the integrated flux densities at 408 and $1420 \mathrm{MHz}$, and $\alpha=0.51 \pm 0.03$ from the best fit to all published flux densities.

The first detailed study of the neutral hydrogen possibly associated with the SNR is that of Landecker et al. (1980) who used the DRAO Synthesis Telescope (Roger et al. 1973) to obtain H I images of G78.2+2.1 with a spatial resolution of $2^{\prime} \times 3^{\prime}$ and spectral resolution of $2.6 \mathrm{~km} \mathrm{~s}^{-1}$. These authors proposed a 
model in which the SNR progenitor exploded in a slab of material, the brightest radio continuum features occuring where the slab and expanding blast wave intersect. They also identified three high-velocity isolated cloudlets which might have been accelerated by the SNR. They estimated the distance of $\mathrm{G} 78.2+2.1$ as $1.5 \mathrm{kpc}$ on the basis of $\mathrm{H}$ I absorption of the brightest continuum structures. Post-shock H I was also found in the Westerbork Radio Synthesis Telescope observations of Braun \& Strom (1986). Based on H I observations at 2'.4 resolution obtained with the RATAN-600 radio telescope, Gosachinskij (2001) further reported finding a large $\mathrm{H}$ I shell having inner and outer diameters of 55 and $75 \mathrm{pc}$, respectively, for an assumed distance of $1.5 \mathrm{kpc}$, expanding at $\sim 10 \mathrm{~km} \mathrm{~s}^{-1}$ with a systemic velocity of $\sim 3 \mathrm{~km} \mathrm{~s}^{-1}$. The deduced number density and mass in this shell were evaluated at $2.5 \mathrm{~cm}^{-3}$ and $1.3 \times 10^{3} M_{\odot}$ respectively, corresponding to an unperturbed number density $n_{\mathrm{o}} \approx 1.6 \mathrm{~cm}^{-3}$. They concluded that the shell, now in its radiative phase, was produced by the stellar wind of the SNR progenitor.

Lozinskaya et al. (2000) analysed Fabry-Perot H $\alpha$ observations of the brighter parts of the SNR and existing ROSAT X-ray observations and obtained, for the X-ray emitting gas corresponding to the bright shell, a temperature of $(1.6-3.2) \times 10^{7} \mathrm{~K}$, corresponding to a shock velocity $v_{\mathrm{s}}=(1-1.5) \times 10^{3} \mathrm{~km} \mathrm{~s}^{-1}$. Assuming a distance of $1.7 \mathrm{kpc}$, corresponding to a shell radius of $15 \mathrm{pc}$, they deduced an age of (4-6) $\times 10^{3} \mathrm{yr}$ and unperturbed number density $n_{0} \approx(0.15-0.3) \mathrm{cm}^{-3}$ for a remnant still in its adiabatic phase. In their analysis of ASCA X-ray pointings covering G78.2+2.1, Uchiyama et al. (2002) observed widespread emission of a temperature of $\sim 0.6 \mathrm{keV}$ bounded by the bright radio regions with limb-brightened thermal emission with a temperature of $\sim 0.8 \mathrm{keV}$. Interpreting the latter as emission from the immediate post-shock region of the SNR blast wave propagating through the ISM, they deduced a shock velocity of $800 \mathrm{~km} \mathrm{~s}^{-1}$ and adiabatic age of $6600 D_{1.5} \mathrm{yr}$ where $D_{1.5}$ is the distance in units of $1.5 \mathrm{kpc}$. Mavromatakis (2003) obtained optical spectra of some of the bright regions of the SNR and found that they were compatible with a shock wave travelling at a speed of $750 \mathrm{~km} \mathrm{~s}^{-1}$ in an unperturbed ISM of number density $n_{\mathrm{o}} \approx 0.3 \mathrm{~cm}^{-3}$ in which clouds of initial density $20 \mathrm{~cm}^{-3}$ were compressed to $\sim 700 \mathrm{~cm}^{-3}$. An age of $\sim 7000 \mathrm{yr}$ was inferred.

Although it is clear from the above that a considerable body of observations is already available on G78.2+2.1, the new high sensitivity and high dynamic range continuum and $\mathrm{H}$ I images of the CGPS justify a further analysis. In this work, we use the continuum images to better delineate the extent of the SNR and the spatial spectral index variations. For the latter, we use a correlation between IR and radio continuum emission to remove the thermal emission before any spectral index analysis. In addition to the TT-plot method, a tomography technique is also used to study spectral index variations at full angular resolution. We then discuss the possibility that a new faint shell, detected to the north-west of the SNR, is associated with the SNR. Finally we use the high-quality CGPS H I data to identify new features morphologically resembling the SNR and discuss various evolutionary scenarios suggested to explain their kinematics.

\section{Data}

The radio observations presented here, of arcminute resolution in both the continuum and the $21 \mathrm{~cm}$ line of $\mathrm{HI}$, are taken from the CGPS (Taylor et al. 2003). Before assembly into a mosaic, the data for the individual fields are carefully processed to remove artifacts and to obtain the highest possible dynamic range, using the routines described by Willis (1999). Accurate representation of all structures to the largest scales is assured by incorporating data from large single antennas with data from the Synthesis Telescope, after suitable filtering in the Fourier domain. Continuum data are derived from the $1.4 \mathrm{GHz}$ Effelsberg survey (Reich et al. 1997). Single antenna H I data are obtained from a survey of the CGPS region made with the DRAO 26-m Telescope (Higgs \& Tapping 2000). H I data have a velocity resolution of $1.3 \mathrm{~km} \mathrm{~s}^{-1}$ with a channel separation of $0.82 \mathrm{~km} \mathrm{~s}^{-1}$. The rms noise in the images at full resolution is $0.03 \mathrm{~K} T_{\mathrm{B}}$ in the continuum and $3 \mathrm{~K} T_{\mathrm{B}}$ in $\mathrm{HI}$.

\section{Results and analysis}

\subsection{Continuum images}

Figures 1 and 2 show the 1420 and $408 \mathrm{MHz}$ radio continuum images as they were extracted from the CGPS. Point sources, most evident at $1420 \mathrm{MHz}$, are still present. Although the general morphology is nearly perfectly circular, considerable structure can be seen, in particular in the southern part of the SNR. Also departure from circular symmetry is seen in the form of large-scale protrusions along a direction more or less $45^{\circ}$ clockwise from the vertical axis. The $15^{\prime}$ feature near $l=2.75$ is an H II region.

In addition to the non-thermal synchrotron emission from the SNR, thermal emission is also present in this direction and is well traced by the infrared (IR) emission from the dust. Figure 3 shows the IRAS $60 \mu \mathrm{m}$ image of the same field of view at a comparable resolution. The IR observations are the high-resolutionprocessed (HIRES) (Fowler \& Aumann 1994; Cao et al. 1997) IRAS images produced at the Infrared Processing and Analysis Center (IPAC) ${ }^{1}$. The image is the result of 20 iterations of the algorithm, giving an approximate resolution of 0.5. Most notable is the striking absence of the SNR in this image.

We also obtained an image at $8.23 \mu \mathrm{m}$ (Band A) from the Midcourse Space Experiment (MSX) ${ }^{2}$. This image (Fig. 4) has the same general morphology as the previous one but with an angular resolution of 19" (Egan et al. 1999; Price et al. 2001). This band may contain contributions arising from both fine-structure line emission and discrete PAH emission bands as well as continuum emission from dust at $\sim 400 \mathrm{~K}$.

\subsection{Radio continuum spectral index}

\subsubsection{TT-plot from raw CGPS images}

Given the exceptionally high quality of the CGPS images, it is now possible to give improved estimates of the radio spectral index and of its possible variations over the remnant. After removing the point sources which are present over the face of the SNR and convolving the 1420 and $408 \mathrm{MHz}$ images to the same resolution, we constructed a TT-plot, in which the brightness temperature at one frequency is plotted point-by-point against the brightness temperature at the other frequency. The spectral index $\beta$ is directly related to the slope of the straight line fitted by regression, $\beta=-\log$ (slope) $/ \log (1420 / 408)$. The TT-plot method is immune from errors in the zero-level of the individual maps. Note that, if there are two emission components present, this method will compute a spectral index dominated by that

\footnotetext{
1 IPAC is funded by NASA as part of the Infrared Astronomical Satellite (IRAS) extended mission under contract to the Jet Propulsion Laboratory (JPL).

2 IPAC provides access to the MSX and IRAS images through the Infrared Science Archive at http://irsa.ipac. caltech.edu
} 


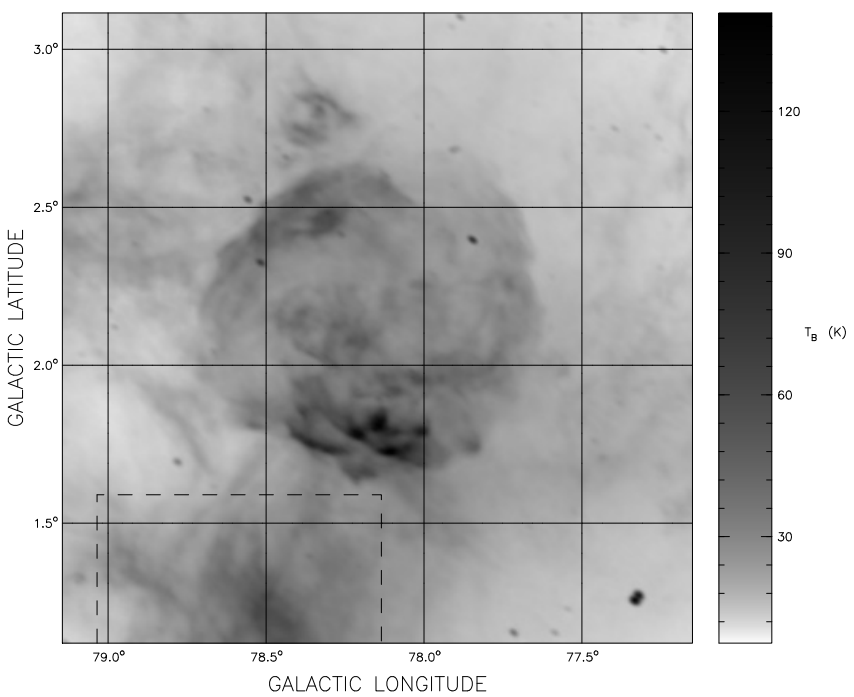

Fig. 1. CGPS radio continuum image of G78.2+2.1 at $1420 \mathrm{MHz}$. Resolution is $0.8 \times 1^{\prime} \cdot 3$. The rectangle in the lower left part of the image shows the northernmost part of the region used to obtain the correlation between IR emission and thermal radio continuum (see text for details). This region extends all the way to a Galactic latitude of 0.5 .

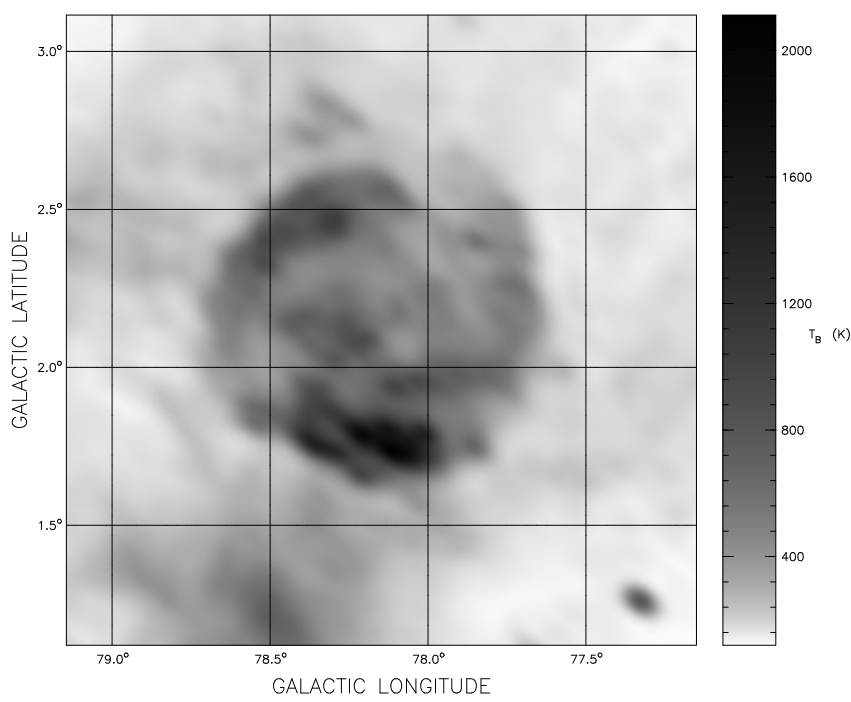

Fig. 2. CGPS radio continuum image of G78.2+2.1 at $408 \mathrm{MHz}$. Resolution is $2.9 \times 4$. 5 .

of the component whose brightness is spatially varying most rapidly.

Figure 5 shows the result of this exercise. A significant amount of scatter is present on the diagram, which does not really come as a surprise given the presence of thermal emission superposed on the non-thermal synchrotron radiation (Wendker et al. 1991) as well as actual spatial variations (Zhang et al. 1997). A technique often used to remove a background consists in fitting a twisted plane to the emission outside the boundary of the object. However G78.2+2.1 is a very large remnant and any variation of this background inside the boundary will introduce a scatter.

\subsubsection{Estimating the thermal radio continuum emission}

It has been shown by a number of authors (e.g., Broadbent et al. 1989; Fürst \& Reich 1987; Moon \& Koo 1994) that, for

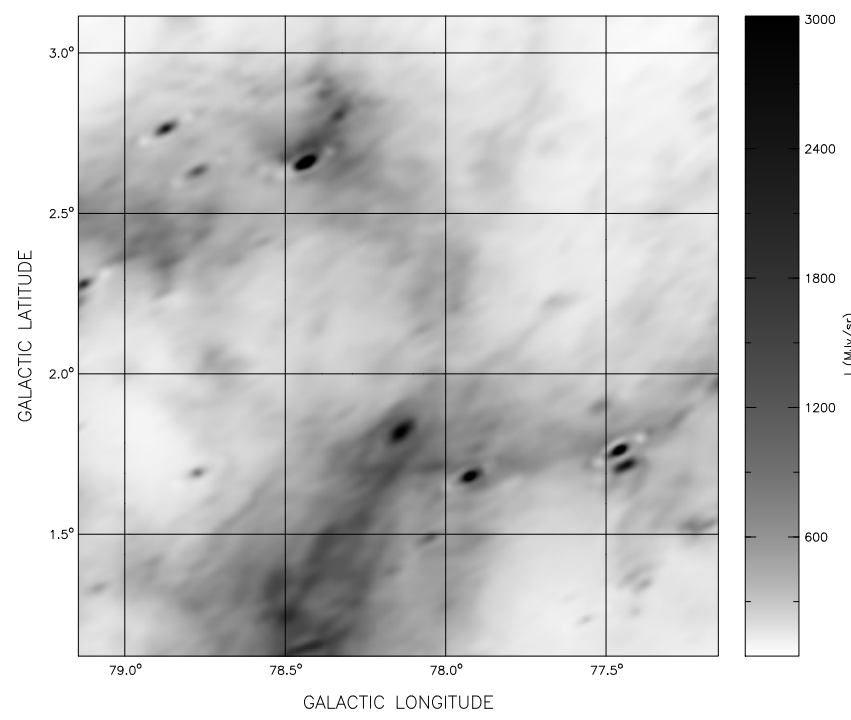

Fig. 3. HIRES $60 \mu \mathrm{m}$ emission of the same area as in the preceding radio continuum images. Resolution is $1^{\prime} .0 \times 1$ '.7.

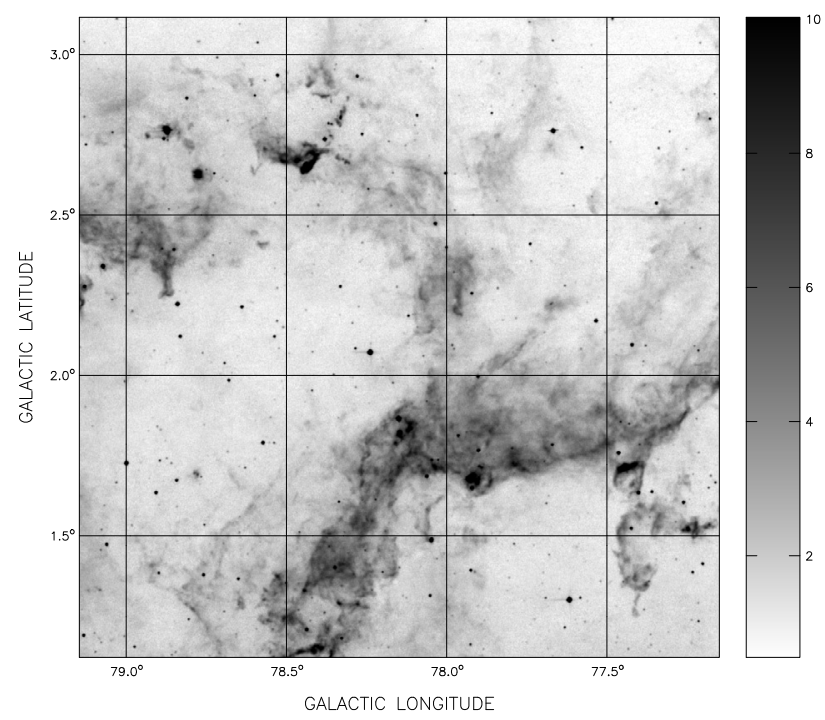

Fig. 4. MSX image at $8.23 \mu \mathrm{m}$ (band A). Resolution is 0.3.

$\mathrm{H}$ II regions, a strong correlation exists between the brightness temperature at $11 \mathrm{~cm}, T_{\mathrm{B}, 11}$, and $I_{60}$, its equivalent at $60 \mu \mathrm{m}$. In particular Broadbent et al. (1989) obtained the relation $T_{\mathrm{B}, 11}=(6.4 \pm 1.7) \times 10^{-3} I_{60}$, where $T_{\mathrm{B}, 11}$ is in $\mathrm{K}$ and $I_{60}$ in $\mathrm{MJy} \mathrm{sr}^{-1}$. Assuming a spectral index $\alpha=-0.10$, one obtains $T_{\mathrm{b}, 11}=0.26 \times T_{\mathrm{b}, 21}$ so that the appropriate relation at $21 \mathrm{~cm}$ becomes $T_{\mathrm{B}, 21}=0.0246 I_{60}$ (Cichowolski et al. 2001) or equivalently $I_{60}=(40.7 \pm 10.7) \times T_{\mathrm{B}, 21}$.

In order to obtain this correlation, we first identified a region to the south of the SNR likely to contain only thermal emission (the northern part of this region is shown by a dashed line in Fig. 1). A TT-plot at 1420 and $408 \mathrm{MHz}$ of this region yielded a value of the temperature spectral index $\beta=2.10 \pm 0.02$ confirming the thermal nature of the emission. We then convolved the $1420 \mathrm{MHz}$ and $60 \mu \mathrm{m}$ images to a common resolution and plotted the brightness at $60 \mu \mathrm{m}\left(I_{60}\right)$ against that at $1420 \mathrm{MHz}$ $\left(T_{\mathrm{B}, 21}\right)$. Regions brighter than $1300 \mathrm{MJy} / \mathrm{sr}$ in the IR were excluded from the analysis as they are likely more compact regions of the ISM. The result is shown in Fig. 6 from which we find 


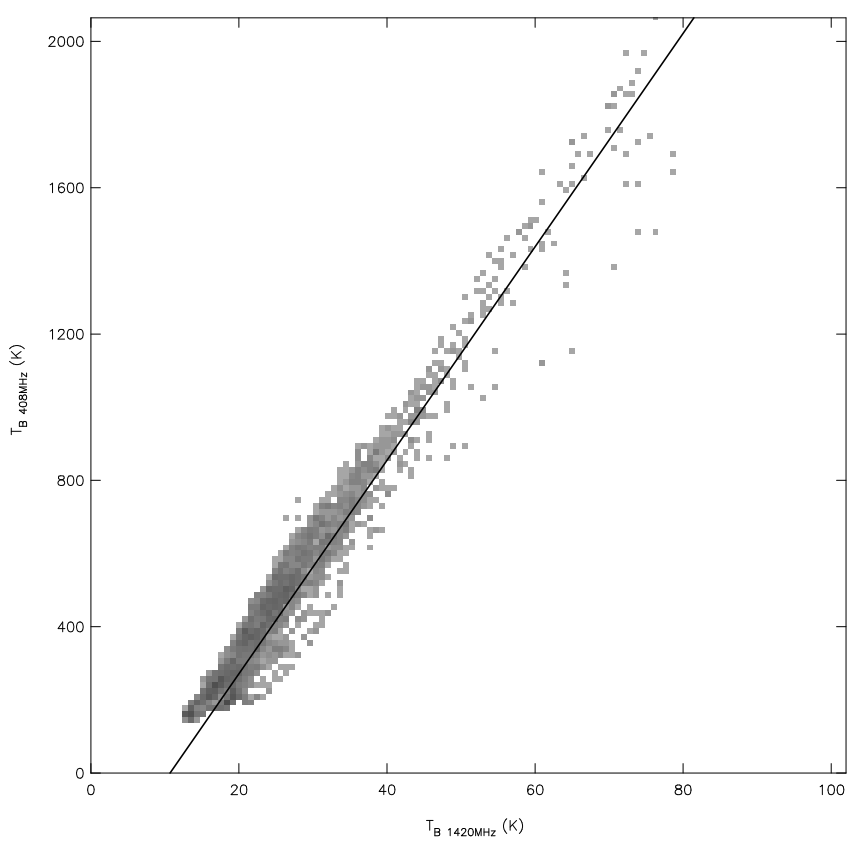

Fig. 5. TT-plot obtained by a pixel-to-pixel comparison of the two CGPS images, after removal of the point sources. See text for details.

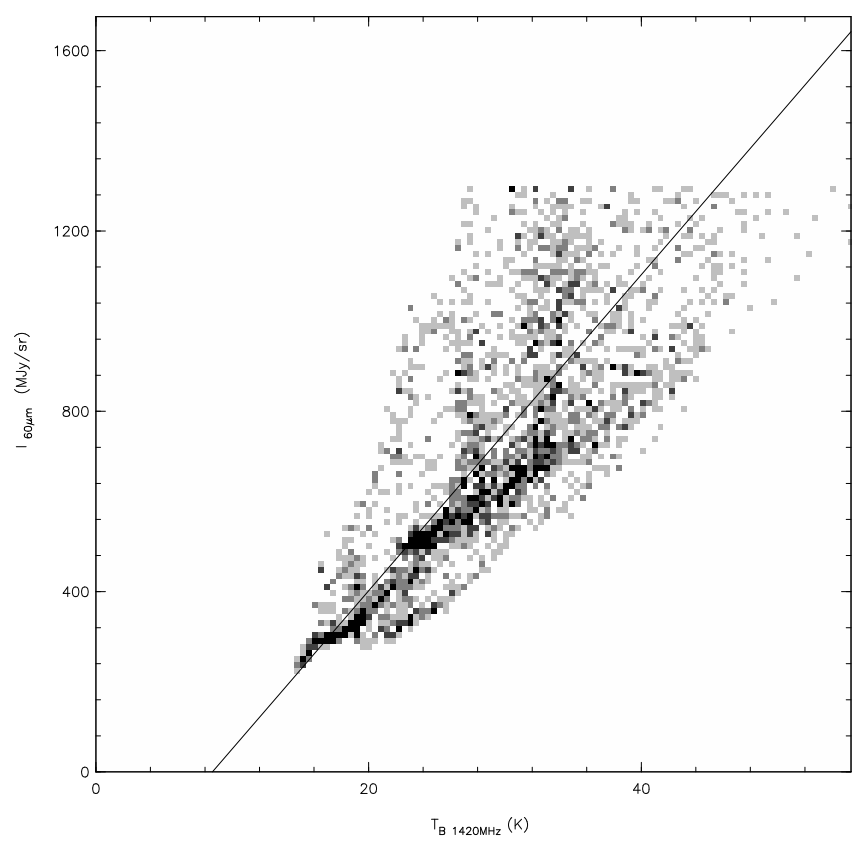

Fig. 6. Plot of the brightness $I_{60}$ at $60 \mu \mathrm{m}$ against the brightness temperature $T_{\mathrm{B}}$ at $1420 \mathrm{MHz}$. The best-fit straight line corresponds to a slope of 0.0285 . Regions brighter than $1300 \mathrm{MJy} / \mathrm{sr}$ were excluded from the analysis.

a slope of 0.0285 , comparable to that obtained by Cichowolski et al. (2001).

Under the assumption that this relationship holds as well within the SNR area, we can then obtain a "theoretical" thermal image at $1420 \mathrm{MHz}$, as shown in Fig. 7. The "theoretical" thermal image at $408 \mathrm{MHz}$ is obtained from the one at $1420 \mathrm{MHz}$ by simply assuming a spectral index $\beta=2.10$ and smoothing the image to the $408 \mathrm{MHz}$ resolution.

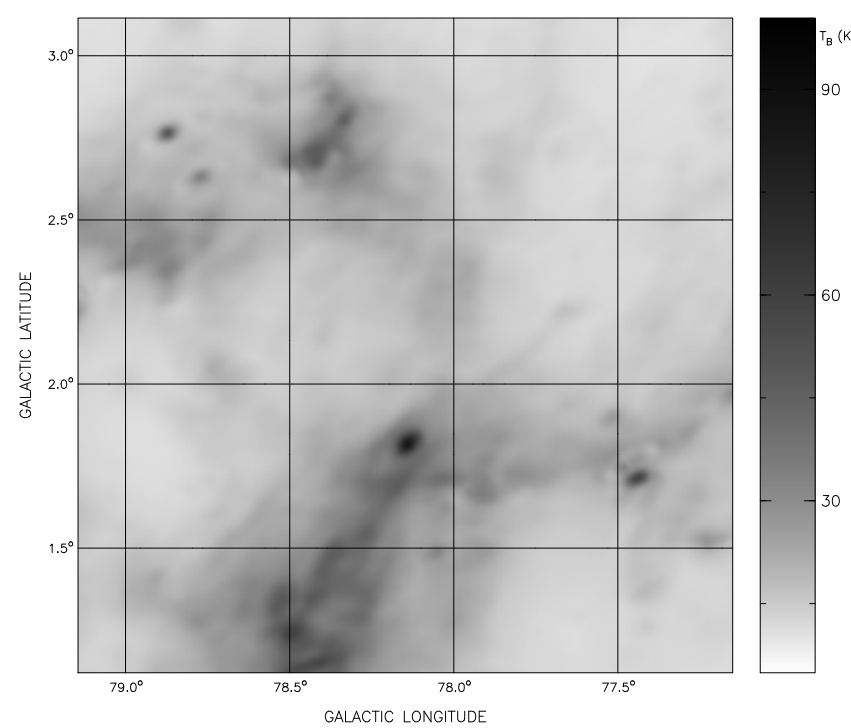

Fig. 7. Theoretical thermal emission at $1420 \mathrm{MHz}$ obtained by applying the correlation between IR and radio continuum brightnesses. See text for details.

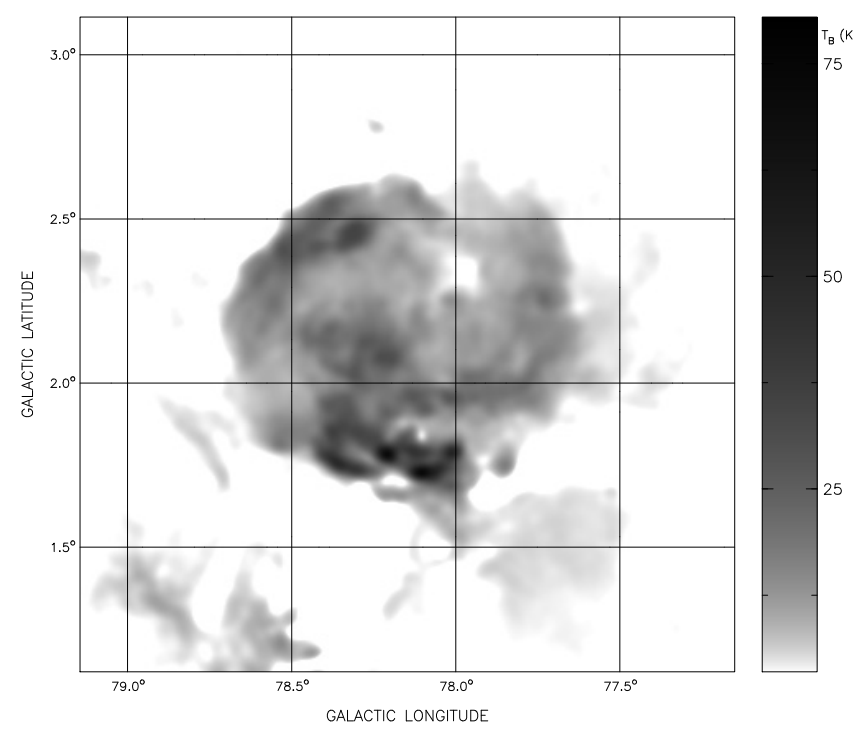

Fig. 8. Non-thermal emission at $1420 \mathrm{MHz}$. Regions were the brightness temperature is smaller than $3.5 \mathrm{~K}$ are white.

\subsubsection{Spectral index determination}

We are now in a position to isolate the non-thermal emission by simply subtracting the "theoretical" thermal images from the corresponding CGPS raw images at both radio frequencies. These pure non-thermal emission images are shown in Figs. 8 and 9.

These non-thermal images were then used to produce new TT-plots. The different regions for which plots were obtained are sketched in Fig. 10 and the TT-plots are shown in Fig. 11. Not surprisingly, the scatter on any of these plots is significantly reduced compared to what it was on the TT-plot obtained with the raw CGPS images. Also many of the deduced spectral indices are steeper than previous determinations (e.g., Zhang et al. 1997). It is interesting that the steeper and lower values of spectral index are found at opposite ends of the SNR, namely to the south and north-northwest respectively. 


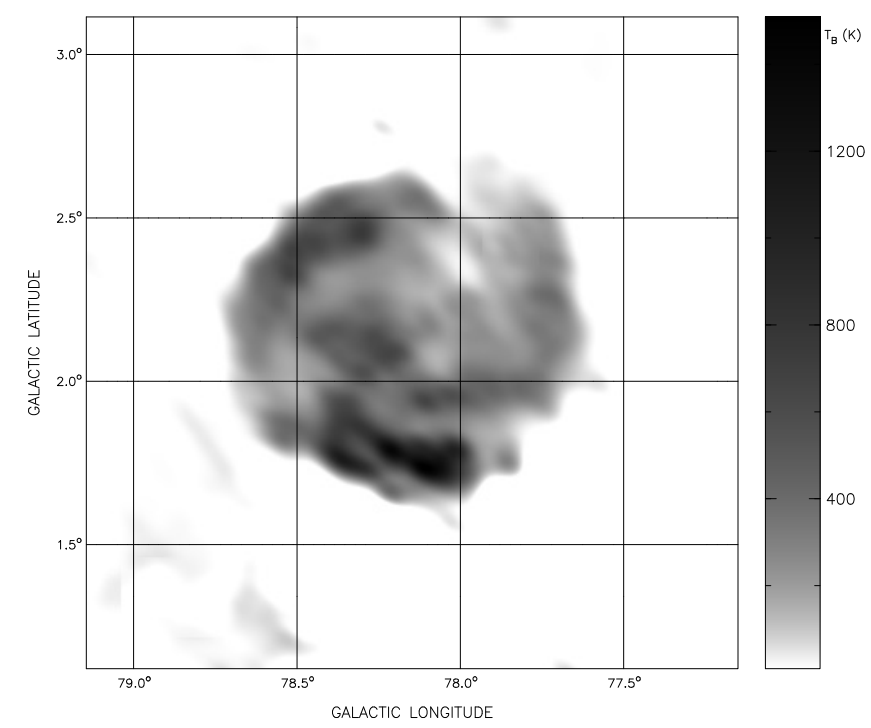

Fig. 9. Non-thermal emission at $408 \mathrm{MHz}$. Regions were the brightness temperature is smaller than $10 \mathrm{~K}$ are white.

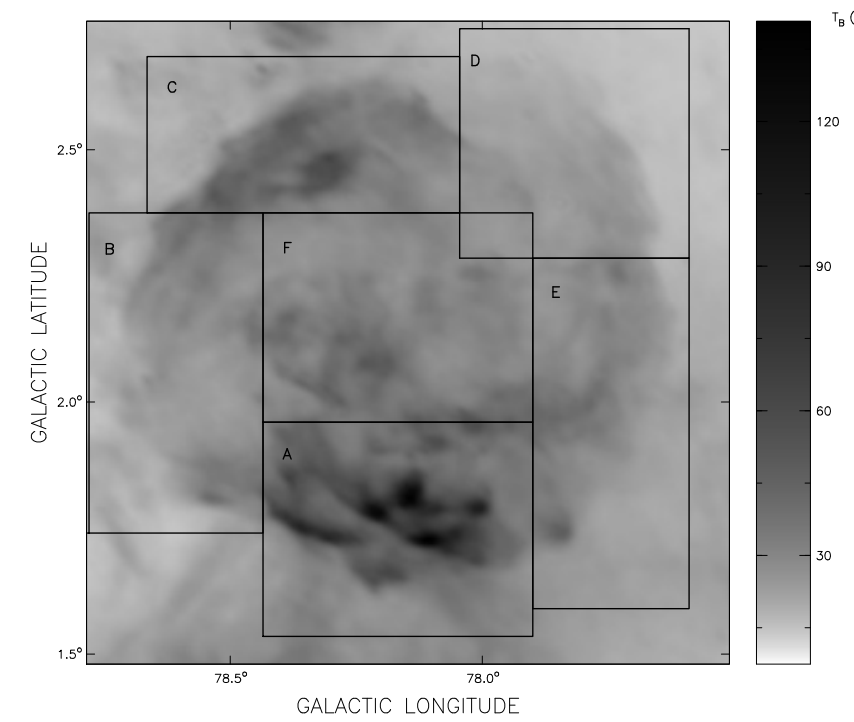

Fig. 10. A $1420 \mathrm{MHz}$ image showing the various regions for which TT-plots were obtained.

In Table 1, we compare these spectral indices with those obtained with the raw CGPS data, i.e., before subtraction of the thermal component. Not surprisingly, a substantial change in $\beta$ is seen for the SNR as a whole. As for the sub-regions, region A shows the largest variation. This region contains the so-called $\gamma$-Cygni Nebula which is a known (Higgs et al. 1977b; Wendker et al. 1991) complication factor for the spectral index determination in this area.

We now proceed to obtain an image of the spectral index variations across the SNR. The first method used, the "convolution differential spectral index" technique, is described in detail by Zhang et al. (1997). It consists in calculating a spectral index from a TT-plot within an analysis box of a given size, and sliding this aperture over the full extent of the input images. At the same time, the error in each fit is computed point-by-point; this error "map" is an essential tool for assessing the value of the spectral index map. The aperture size was chosen as a compromise between minimizing errors while retaining the ability to detect small-scale spectral variations. The aperture must be large enough that there is significant brightness variation within it.

As a first step and in order to compare our results to those of Zhang et al. (1997), we used exactly the same parameters with the raw CGPS images from which only point sources had been subtracted. A region centered on the $\gamma$-Cygni nebula and a strong extragalactic point source, which could not be removed at $408 \mathrm{MHz}$, were excluded. The images were convolved to a resolution of 6. $0 \times 4.4$ and an analysis box four times the size of the beam was used. The result of this analysis is shown in Fig. 12, where the image has been converted to equatorial coordinates (B1950) in order to compare with the results of Zhang et al. (1997). If we compare our results (Fig. 12) with Fig. 13 taken from Zhang et al. (1997), we can note some similarities as well as differences. Both analyses show that there are significant variations across the SNR. A major difference is that our spectral index values are systematically higher than those of Zhang et al. (1997) and that, except for one region, the larger values of spectral index are near the periphery of the SNR. A "tongue" of steep spectral index, seen running north-east to south-west in the north-eastern half of the SNR on the image of Zhang et al. (1997) is virtually absent on our image. The higher quality CGPS images have allowed us to map a region of weaker spectral index to the north-west of the SNR.

Because the CGPS data are of higher resolution and better sensitivity than the ones used in the analysis of Zhang et al. (1997), we have repeated the procedure, but using this time the CGPS images from which both point sources and the thermal component had been removed. The images were convolved to a common resolution of $4.5 \times 2$ '.9 and an analysis box of four times the beam size was used. Only a region centered on the previously mentioned strong extragalactic point source needed to be excluded since the thermal subtraction procedure did remove most of the emission from the $\gamma$-Cygni Nebula. The result is shown in Fig. 14 (note that we have now reverted to using Galactic coordinates).

A region of steeper spectral index seems to run roughly vertically through the SNR, being more extended in the south-west. It seems that the eastern part of the remnant shows relatively weak gradients in spectral index while the western side is characterized by stronger gradients.

The previous method of spectral index determination is rather robust, however it sacrifices resolution. Another method, devised by Katz-Stone \& Rudnick (1997) in their study of the spectral index variations in some radio galaxies, does not suffer from this limitation. Called "tomography", the technique consists in creating a series of images " $i$ " thus obtained:

$T_{i}\left(\beta_{i}\right)=T_{\mathrm{B} 408}-T_{\mathrm{B} 1420}\left(\frac{408}{1420}\right)^{-\beta_{i}}$

where $T_{\mathrm{B} 408}$ and $T_{\mathrm{B} 1420}$ are the observed images at 408 and $1420 \mathrm{MHz}$, respectively. The second term on the right of Eq. (1) is a $408 \mathrm{MHz}$ image obtained from the $1420 \mathrm{MHz}$ image, assuming a spectral index $\beta_{i}$. If the true spectral index $\beta$ is $\beta_{i}$ over the entire image, then the image corresponding to $T_{i}\left(\beta_{i}\right)$ is identically blank (all pixels have value 0.0 ). In other words, structures having spectral index $\beta_{i}$ are absent from image $i$, whereas a structure having a lower (higher) value of spectral index will be characterized by a negative (positive) temperature $T_{i}$. 

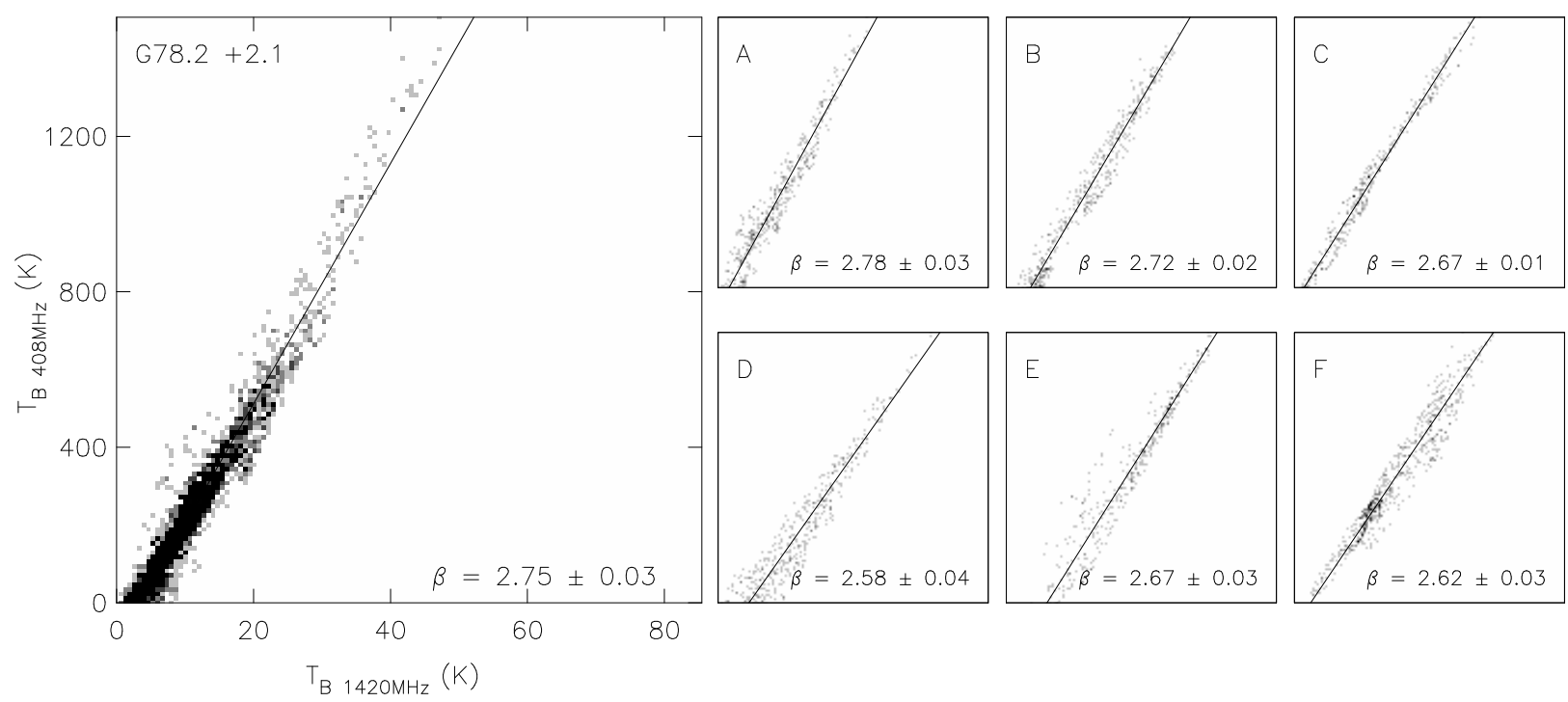

Fig. 11. TT-plots taken over the entire SNR (larger plot on the left) and over the various regions defined in the previous figure.

Table 1. Brightness temperature spectral index $\beta$ before and after subtraction of the thermal component.

\begin{tabular}{ccc}
\hline \hline Region & Before & After \\
\hline Entire SNR & $2.59 \pm 0.03$ & $2.75 \pm 0.03$ \\
A & $2.66 \pm 0.02$ & $2.78 \pm 0.03$ \\
B & $2.70 \pm 0.03$ & $2.72 \pm 0.02$ \\
C & $2.65 \pm 0.01$ & $2.67 \pm 0.01$ \\
D & $2.50 \pm 0.05$ & $2.58 \pm 0.04$ \\
E & $2.72 \pm 0.05$ & $2.67 \pm 0.03$ \\
F & $2.64 \pm 0.05$ & $2.62 \pm 0.03$ \\
\hline
\end{tabular}

It is necessary, or at least preferable, to remove any thermal contribution, since any such emission will bias the results. Indeed Eq. (1) becomes with two components

$$
\begin{aligned}
T_{i}\left(\beta_{i}\right)=\left[T_{\mathrm{B}(408)_{\mathrm{t}}}+T_{\mathrm{B}(408)_{\mathrm{nt}}}\right]-\left[T_{\mathrm{B}(1420)_{\mathrm{t}}}+T_{\mathrm{B}(1420)_{\mathrm{nt}}}\right]\left(\frac{408}{1420}\right)^{-\beta_{i}}, \\
T_{i}\left(\beta_{i}\right)=\left[T_{\mathrm{B}(408)_{\mathrm{t}}}-T_{\mathrm{B}(1420)_{\mathrm{t}}}\left(\frac{408}{1420}\right)^{-\beta_{i}}\right] \\
+\left[T_{\mathrm{B}(408)_{\mathrm{nt}}}-T_{\mathrm{B}(1420)_{\mathrm{nt}}}\left(\frac{408}{1420}\right)^{-\beta_{i}}\right],
\end{aligned}
$$

where subscripts $\mathrm{t}$ and $\mathrm{nt}$ refer to the thermal and non-thermal components, respectively. If thermal emission is negligible, the first term between square brackets on the right-hand side of Eq. (3) is negligible.

Figure 15 reproduces a gallery of images obtained by varying $\beta$ from 2.20 to 2.75 and where the absolute value of each pixel is displayed. Since the thermal emission has been subtracted, the first panel $(\beta=2.20)$ reproduces quite well the actual morphology of the SNR (the emission from the SNR being non-thermal, there are no regions with such a low spectral index, hence the pixels all have absolute values much larger than 0 ). This figure does not lend itself to a simple interpretation, however it does convey the general impression that significant variations are indeed present. For instance the north-western sector shows relatively smaller values of spectral index than the north-eastern sector. Furthermore a series of structures in the extreme south are

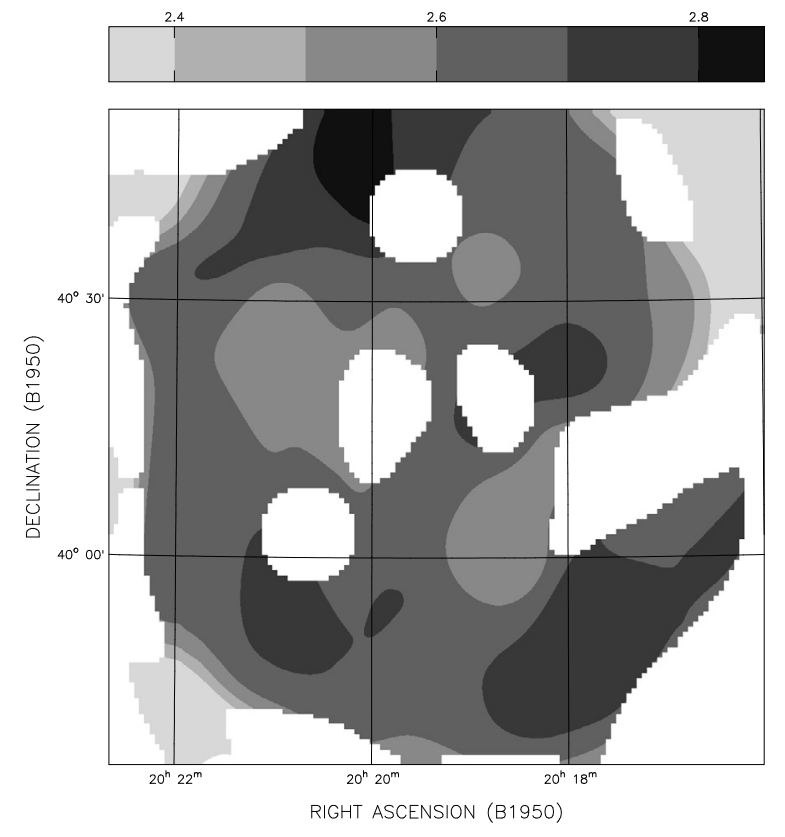

Fig. 12. Low-resolution spectral index map from raw CGPS images. Regions where the error exceeds 0.06 have been blanked out. The two circular white regions excluded from the analysis correspond to the $\gamma$-Cygni Nebula and a strong extragalactic point source (see text). Note that this image is in equatorial coordinates.

characterized by values larger than 2.70 , as was already noted before. Two relatively conspicuous and roughly circular regions are present between 2.60 and 2.75 in the north-west and northeast and are probably extragalactic (discussed later).

From the separate gallery images of Fig. 15, it is possible (and desirable) to obtain a single image by assigning to each pixel the value $\beta_{i}$ that gives that pixel the value closest to 0 . A more detailed analysis of the method will be given elsewhere (Ladouceur \& Pineault, in preparation). For the sake of illustration, we present here an image, Fig. 16, obtained by choosing the value $\beta_{i}$ of each pixel according to the following formula:

$\beta_{i}=\frac{\sum_{i=1}^{N} \beta_{i} / T_{i}\left(\beta_{i}\right)^{2}}{\sum_{i=1}^{N} 1 / T_{i}\left(\beta_{i}\right)^{2}}$. 


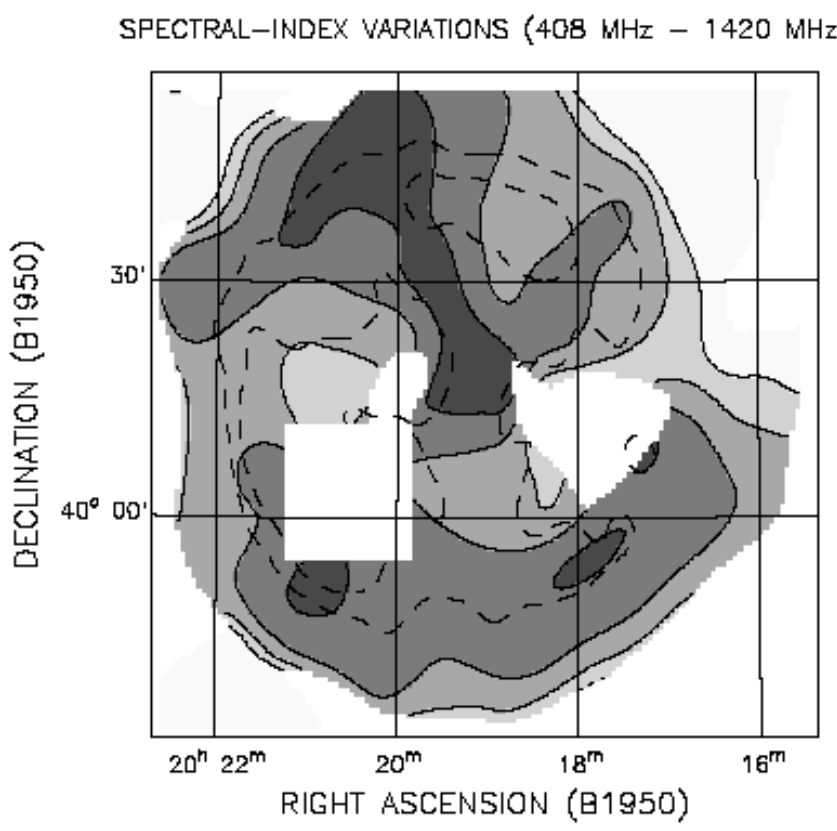

Fig. 13. Low-resolution spectral index map from Zhang et al. (1997), reproduced with permission from A\&A. Regions where the error exceeds 0.06 have been blanked out. The white square marks the position of the $\gamma$-Cygni Nebula, excluded from the analysis. The shading values are the same as in the previous figure. Note that this image is in equatorial coordinates.

This figure confirms and summarizes our view on the observed spectral index variations over the SNR. The two regions at about 2.3 with the steepest spectral index (near $77^{\circ} .8$ and 78.5) are believed to be extragalactic, as we discuss further on the basis of $\mathrm{HI}$ absorption profiles. Among the small-scale structures seen to have a steeper spectral index in the south-west, a bullet-like feature near $l \approx 77.85, l \approx 1.75$ could represent the location of a fast-moving clump of ejecta.

\subsection{Neutral hydrogen morphologically related to the SNR}

The CGPS observations cover a velocity range between +58 and $-152 \mathrm{~km} \mathrm{~s}^{-1}$ (all H I velocities are with respect to the local standard of rest - LSR). Figure 17 shows the average H I spectrum in a circular area of diameter $100^{\prime}$ around the position of G78.2+2.1. The most notable features are the local arm (from about +20 to $-20 \mathrm{~km} \mathrm{~s}^{-1}$ ), the Perseus arm (from -20 to $-50 \mathrm{~km} \mathrm{~s}^{-1}$ ), and the outer arm (from -50 to $-120 \mathrm{~km} \mathrm{~s}^{-1}$ ). Figure 18 shows the Galactic rotation curve along the same line of sight, required to transform an LSR velocity into a distance. The empirical curve of Brand \& Blitz (1993) for a number of Galactic longitudes as well as the theoretical relation for a flat rotation curve, with a circular velocity $V_{\mathrm{o}}=220 \mathrm{~km} \mathrm{~s}^{-1}$ at a Solar Galactocentric distance $R_{\mathrm{O}}=8.5 \mathrm{kpc}$, are presented. It is clear from this figure that, in addition to the inherent spatial confusion due to the fact that we are looking basically along a spiral arm, velocity crowding can be extremely severe especially for distances less than about $4 \mathrm{kpc}$.

The resolution and sensitivity of the CGPS images being considerably better than the ones that were available to Landecker et al. (1980), we have inspected the entire data cube looking for structures morphologically related to the SNR. For ease of presentation, $\mathrm{HI}$ images have had a baseline subtracted equal to the average $\mathrm{H}$ I level in a $100^{\prime}$ diameter central region in

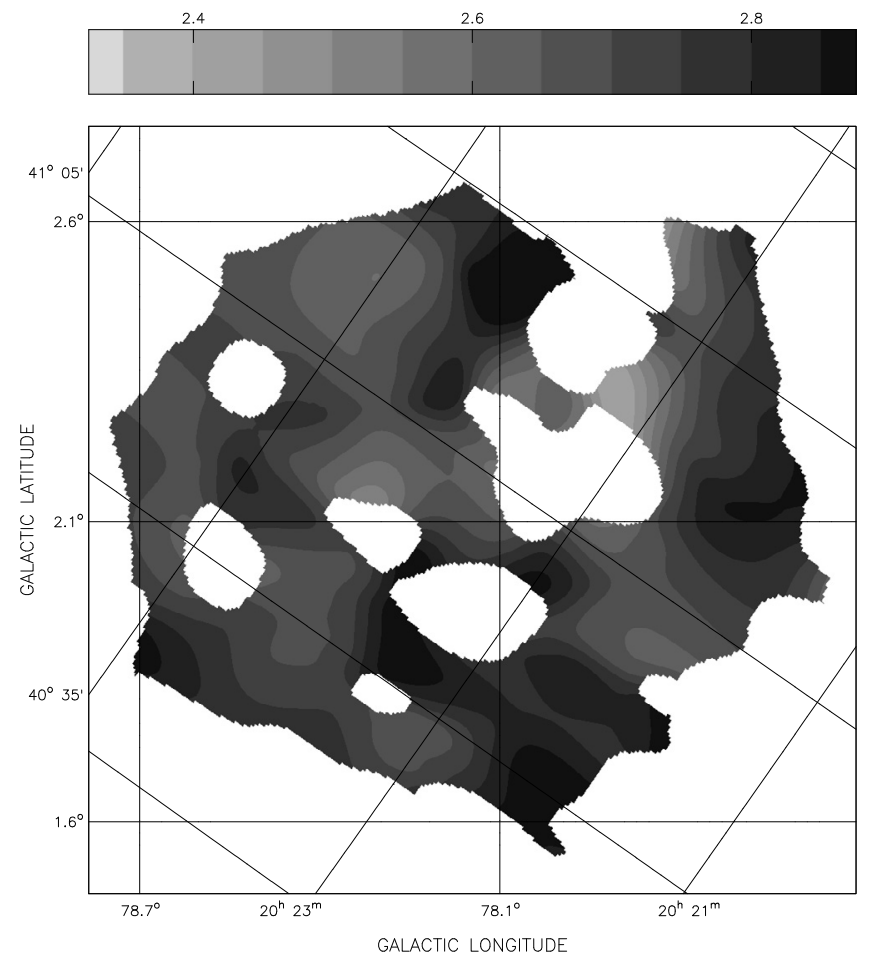

Fig. 14. High-resolution spectral index map from the non-thermal CGPS images. Regions where the error exceeds 0.06 have been blanked out. The circular white region corresponds to the excluded extragalactic point source. Note that this image is in Galactic coordinates.

that velocity range. This does not distort the H I structure in the images, but simply reduces the dynamic range required for their display. The amount subtracted from each image can be read off the spectrum of Fig. 17.

As already pointed out by Landecker et al. (1980) and Pineault et al. (2008), considerable absorption against the brightest parts of the SNR is present. We have made an average of the relevant CGPS channel images and present the result in Fig. 19. Figure 20 is a $1420 \mathrm{MHz}$ continuum image on which all features over which an absorption profile was obtained are identified. Sources A, E and G show absorption all the way to about $-80 \mathrm{~km} \mathrm{~s}^{-1}$ and are presumed extragalactic. All other compact features show no absorption at velocities more negative than $-12 \mathrm{~km} \mathrm{~s}^{-1}$ and are thus considered Galactic and most likely associated with the SNR.

The simplest expected signature of an SNR observed in H I is that of a cavity possibly surrounded by a denser shell of matter. Background emission may however "fill in" this minimum and associated structures may be quite difficult to identify especially, as is the case here, if one is looking along a spiral arm. We have nevertheless identified two features which we believe have a reasonable chance of being associated with the SNR. The first one, shown in Fig. 21, which is an average over velocities between 9 and $3 \mathrm{~km} \mathrm{~s}^{-1}$, is a partial shell surrounding the SNR from north through south-east to south-west. It is absent in the northwest. The location of this shell is nearly coincident with a similar structure proposed by Gosachinskij (2001). Figure 22 shows that the bright radio continuum emission is indeed circumscribed by the H I feature. We have also identified a second partial H I shell in the velocity range between -11 and $-19 \mathrm{~km} \mathrm{~s}^{-1}$. Figure 23 shows that this shell completes the previous shell in the northwestern quadrant, both shells circumscribing completely the radio continuum. 


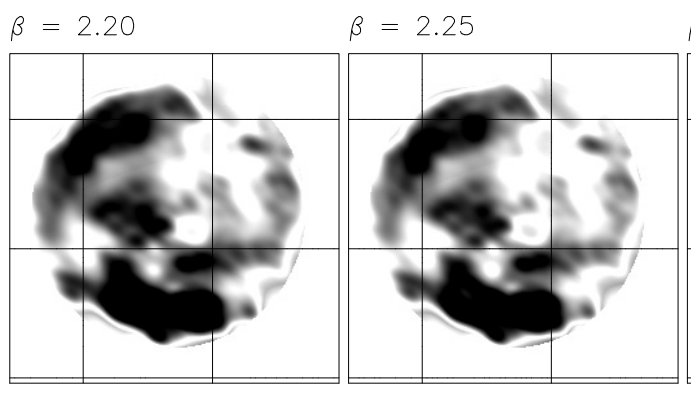

$\beta=2.30 \quad \beta=2.35$

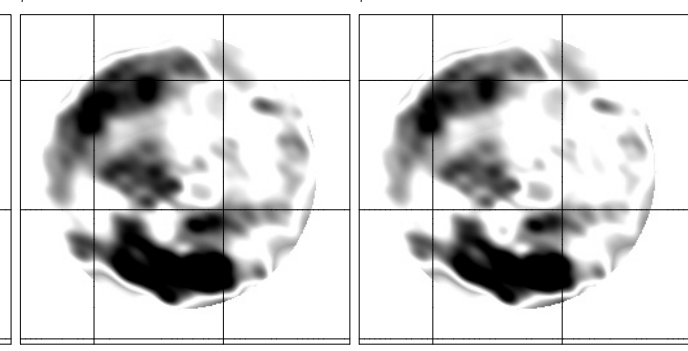

$\beta=2.40$

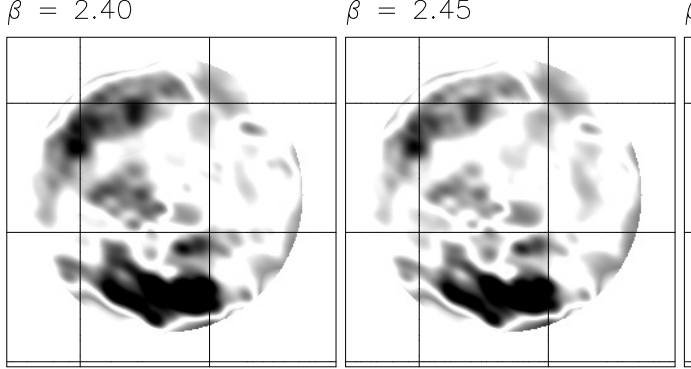

$\beta=2.50$

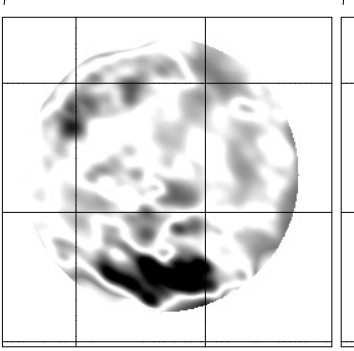

$\beta=2.55$

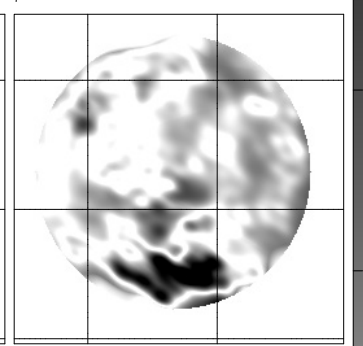

$T_{B}(K)$

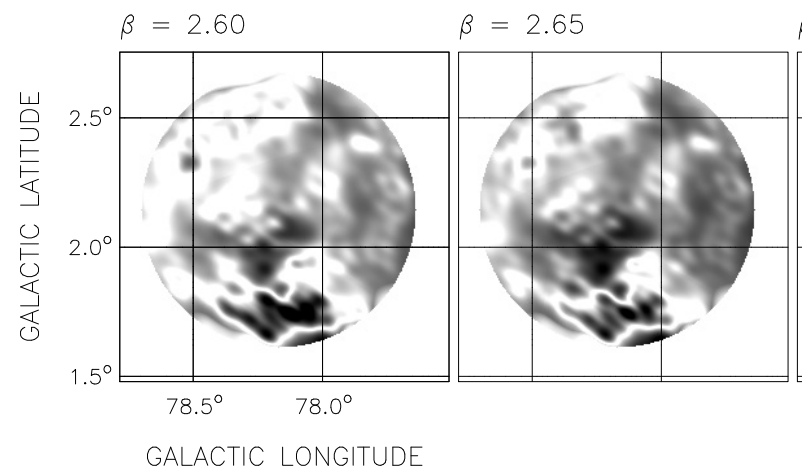
$\beta=2.70$ $\beta=2.75$
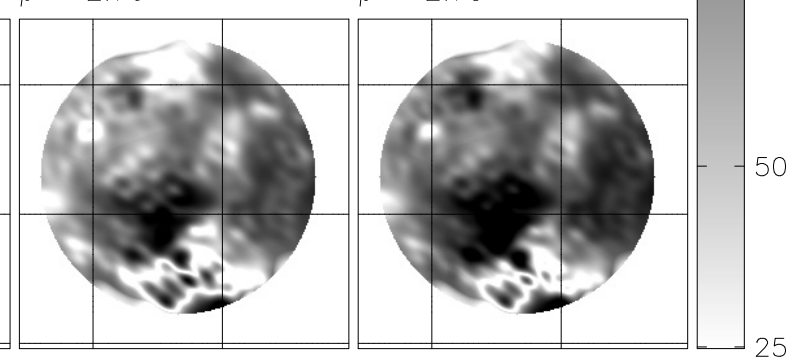

GALACTIC LONGITUDE

Fig. 15. A gallery of images obtained by applying Eq. (1) with values of $\beta_{i}$ ( $\beta$ in this figure) ranging from 2.20 to 2.75.

We also inspected the $\mathrm{CO}$ images of this region obtained by Leung \& Thaddeus (1992). Figure 24 displays an average of the emission between 3 and $10 \mathrm{~km} \mathrm{~s}^{-1}$, showing a morphology similar to that of the first H I shell (Fig. 21), in particular the lack of significant emission to the north-west. An average over the same channels as the second H I shell (Fig. 23) fails however to show a $\mathrm{CO}$ counterpart to the north-western $\mathrm{H}$ I shell.

\subsection{Extended emission to the north-west}

Given the relatively high dynamic range of the CGPS data, we paid extra attention to the weaker emission surrounding the SNR, in particular in the north-west. We have found evidence of what appears to be a nearly complete shell nearly $1^{\circ}$ in diameter. This structure is seen in the radio continuum at 1420 and $408 \mathrm{MHz}$ (Figs. 25 and 26), in the $21 \mathrm{~cm} \mathrm{HI}$ line (Fig. 27) and in the $60 \mu \mathrm{m}$ image (Fig. 28). Although the projected position of this structure near the north-western periphery of the SNR could be a simple line-of-sight coincidence, the fact that the continuum radio shell in that sector is somewhat fainter and slightly outside the more or less spherical outline of the remnant suggests that this faint shell could have been caused by a breakout of the SNR blast wave into a medium of lower density.

Figure 29 shows an archival ROSAT ${ }^{3} \mathrm{X}$-ray image showing emission between 0.1 and $2.4 \mathrm{keV}$. X-ray emission is seen to coincide with the brightest radio emitting regions of the SNR to the north-east, south and south-west but is absent in the northwest. Figure 30, which is a superposition of the X-ray emission and $1420 \mathrm{MHz}$ continuum image, shows that the distant X-ray emission to the north-west matches closely the outer boundary of the new faint shell (Fig. 25).

\subsection{Other features of potential interest}

In this section, we discuss some features which are potentially of interest, either because they could be associated with the SNR or because they can now be ruled out as being associated.

A feature of interest is a compact, but resolved, feature located at $l \approx 77085, b \approx 1.75$ on the radio continuum image (Fig. 1). This knot of emission, well distinct from other structures, especially at $1420 \mathrm{MHz}$, could mark the position of a clump of fast-moving ejecta accelerated by the SNR blast wave

\footnotetext{
3 http://www.mpe.mpg.de/xray
} 


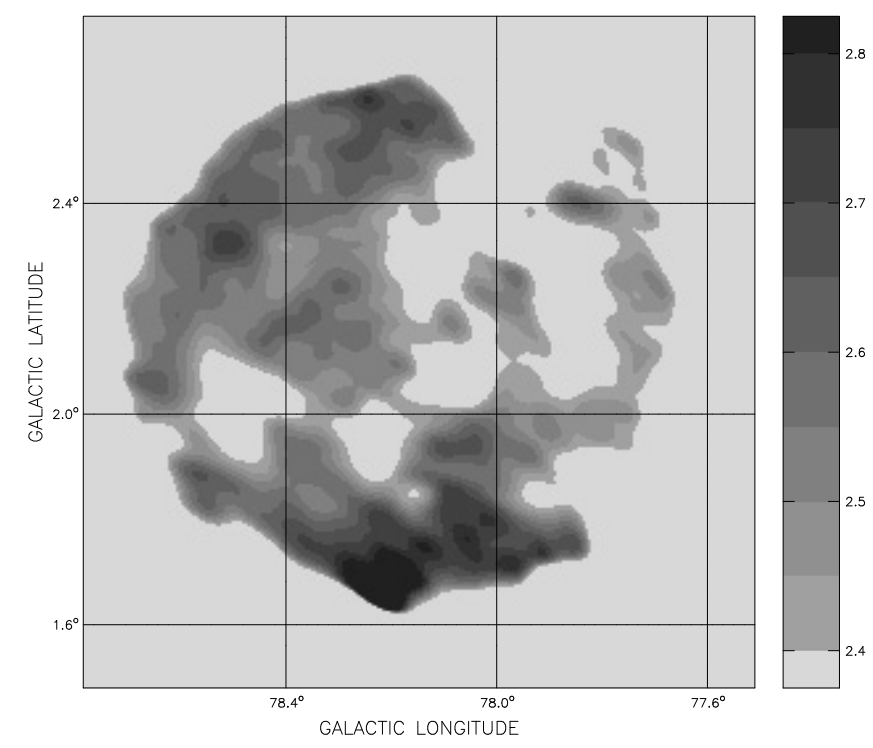

Fig. 16. Spectral index image obtained by combining the separate tomography gallery images into a single image according to Eq. (1).

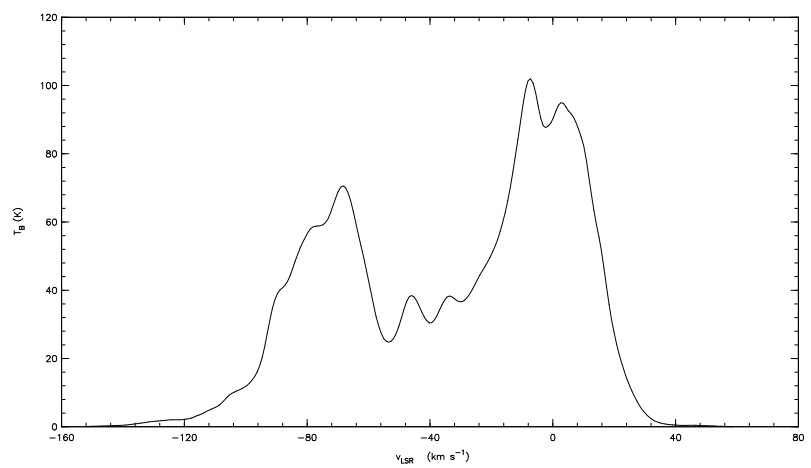

Fig. 17. Average H I spectrum over a region of diameter $100^{\prime}$ centered on the SNR.

and which has caught up with the decelerating shock. Eleven such paraboloidal features or bow shocks have been observed in Cas A (Anderson et al. 1991). Cas A is however a considerably younger remnant than G78.2+2.1. This "bullet" is definitely part of the SNR as observed on the basis of absorption (it is labelled "O" in Fig. 20) and it is also characterized by a steeper value of the temperature spectral index $\beta$ on both spectral index maps (Figs. 14 and 16). There is no H I counterpart at any LSR velocity. It is also not present on the X-ray images of Uchiyama et al. (2002). Figures 31 and 32 also show that the $1420 \mathrm{MHz}$ and high-resolution MSX $8.23 \mu \mathrm{m}$ images exhibit a remarkable correlation.

On the basis of their H I observations, Landecker et al. (1980) had identified three high-velocity isolated cloudlets seen projected within the SNR outline, which they had argued could have been accelerated by the SNR shock wave. Two of these are within the velocity range of the CGPS. From Fig. 33, it is clear that the first of these [cloudlet IIa in Table I of Landecker et al. (1980) - cloudlet IIb is outside the range of sampled CGPS velocities] shows a significant amount of structure, including a clearly defined brighter condensation. It is the only $\mathrm{H}$ I emitting gas present in a $6^{\circ} \times 6^{\circ}$ region in the velocity range from 52 to $45 \mathrm{~km} \mathrm{~s}^{-1}$. This and the fact that it is seen projected well within the remnant make it highly plausible that it is indeed gas accelerated by the SNR shock. It has no obvious counterpart in the radio

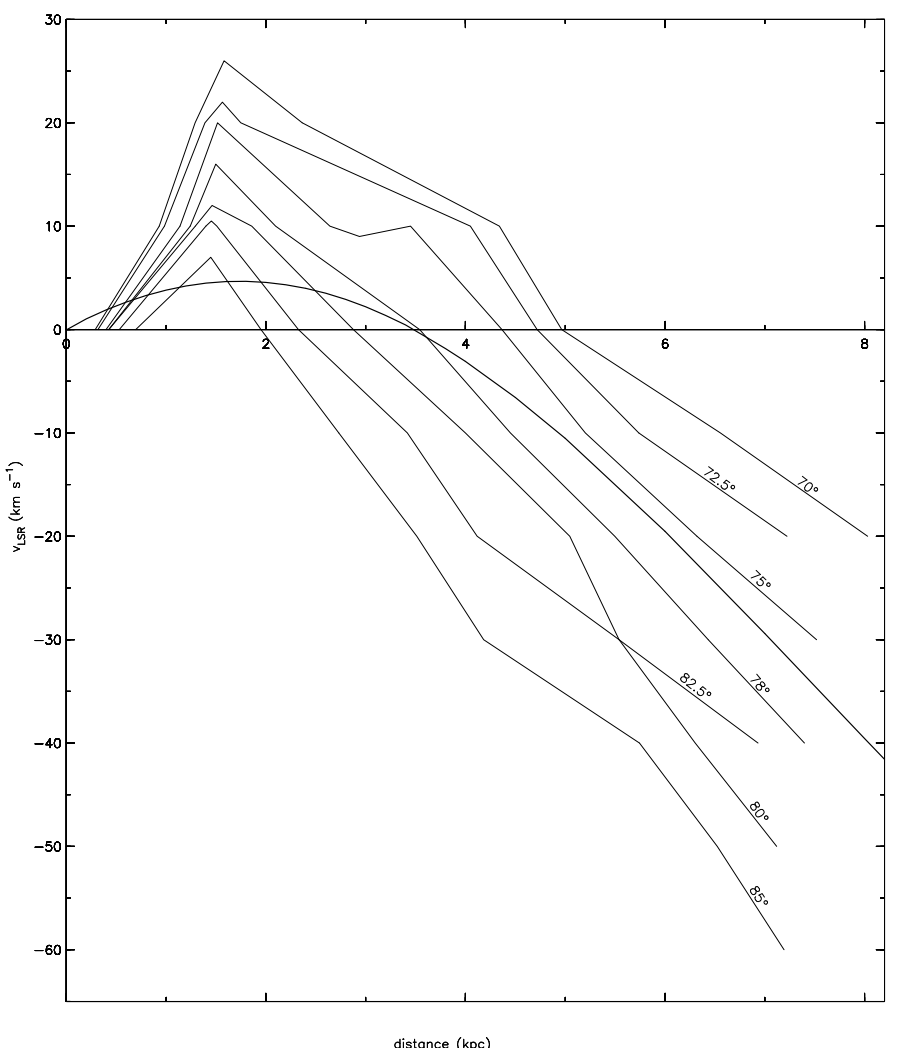

Fig. 18. Variation of kinematic distance as a function of velocity with respect to the LSR. The smooth unlabelled line represents a flat rotation curve model with $R_{\mathrm{o}}=8.5 \mathrm{kpc}$ and $V_{\mathrm{o}}=220 \mathrm{~km} \mathrm{~s}^{-1}$, for the Galactic longitude of G78.2+2.1. Thin ragged lines are empirical curves taken from Brand \& Blitz (1993) and correspond to the given Galactic longitudes.

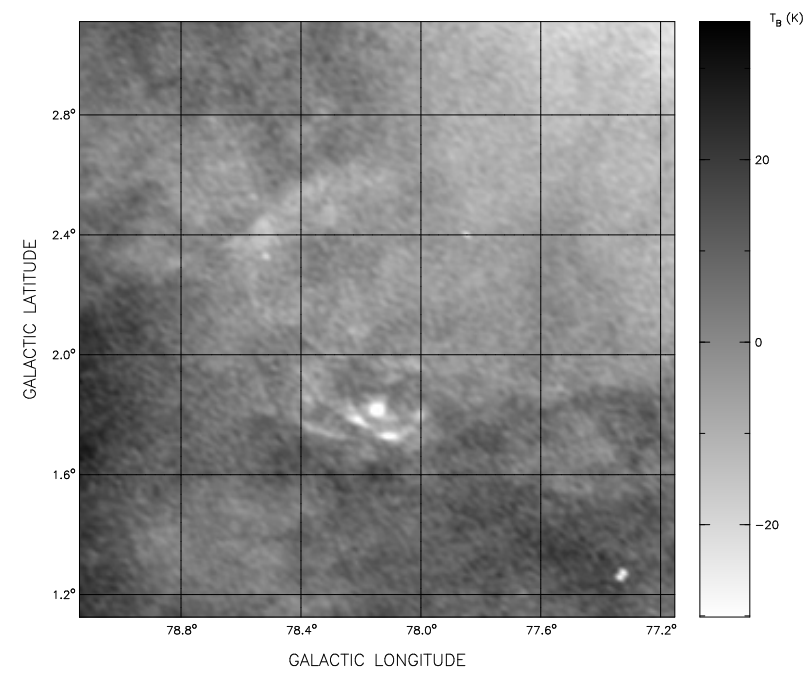

Fig. 19. Average of 19 CGPS channels between 18 and $3.3 \mathrm{~km} \mathrm{~s}^{-1}$ showing the remnant in absorption.

continuum images. We note that it is seen in the same quadrant as the "bullet" described above.

The CGPS H I data show clearly that the second cloudlet proposed by Landecker et al. (1980) at a velocity of $\sim-117 \mathrm{~km} \mathrm{~s}^{-1}$ is part of a larger H I structure connecting with the larger-scale emission to the north. For simplicity, we show only two CGPS channels in Fig. 34, although the connection can be seen from -111 to $-119 \mathrm{~km} \mathrm{~s}^{-1}$. We conclude that this feature is not a 


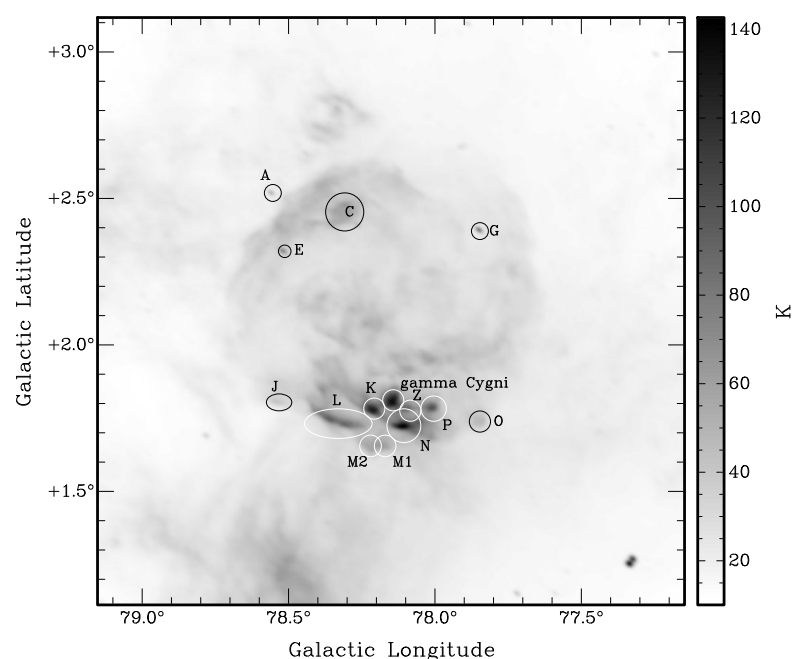

Fig. 20. Identification of structures for which an absorption profile was obtained.

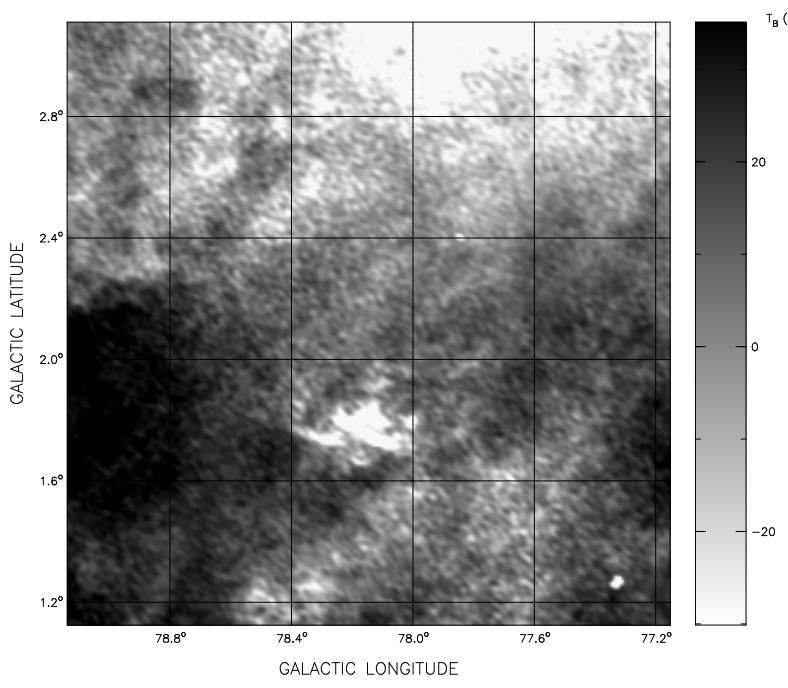

Fig. 21. Average of 9 CGPS channels between 9 and $3 \mathrm{~km} \mathrm{~s}^{-1}$.

cloudlet accelerated by the SNR shock and shall not discuss it further.

Finally Fig. 35 shows the HI emission in channels between -67 and $-70 \mathrm{~km} \mathrm{~s}^{-1}$. A filament is clearly seen starting near $\left(77^{\circ} 9,2^{\circ} .0\right)$ and curving down in a south-eastern direction. As this filament appears projected onto the south-western sector of the SNR, it could be linked to it, although its velocity is very significantly different from the velocities found for the two previous shells. A possible explanation is discussed in the next section.

\section{Discussion}

\subsection{The radio spectral index}

The average value we have obtained for the radio spectral index of the SNR as a whole, $\beta=2.75$, is significantly higher than the one obtained by Zhang et al. (1997) for the same pair of frequencies ( 2.52 between 408 and $1420 \mathrm{MHz}$, with significant spatial variations of \pm 0.15 ). Zhang et al. (1997) observe that their variations may be explained, at least in part, by the presence of thermal emission. Since we have removed most of the thermal emission before evaluating the spectral index, we feel that the variations we detect are intrinsic to the SNR.

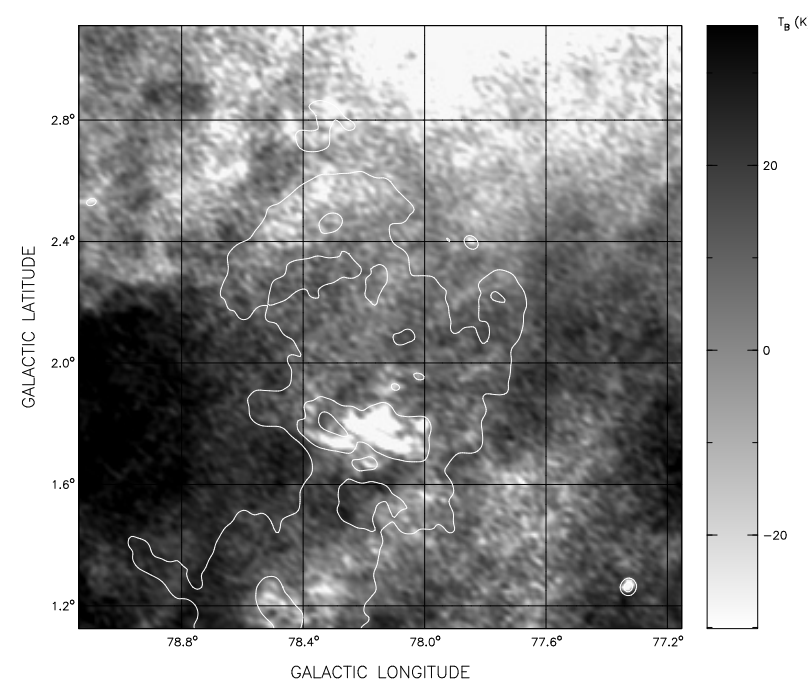

Fig. 22. Same as previous figure but with two $1420 \mathrm{MHz}$ continuum contours at 28 and $50 \mathrm{~K}$.

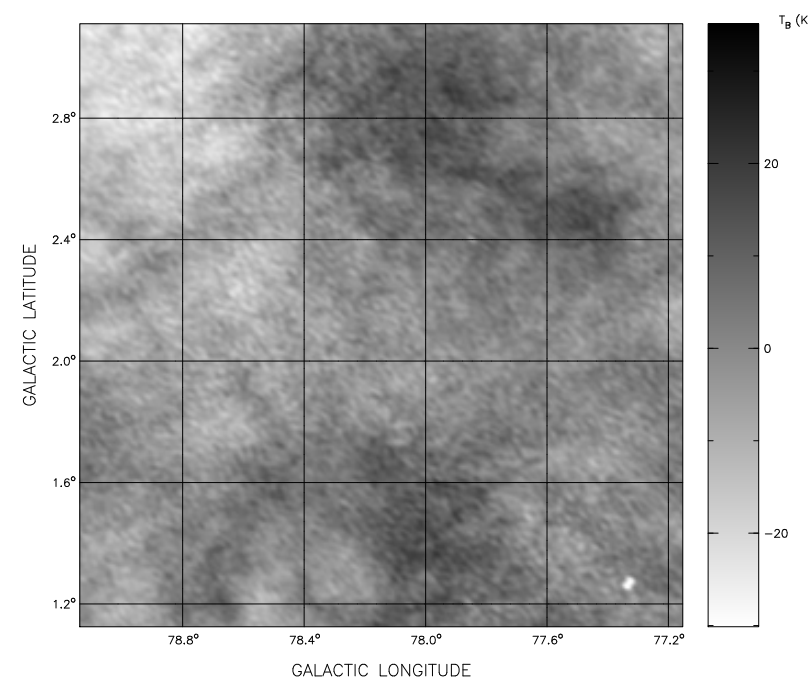

Fig. 23. Average of 10 CGPS channels between -11 and $-19 \mathrm{~km} \mathrm{~s}^{-1}$.

Both the TT-plot method and the tomography technique indicate that these variations in $\alpha$ range from about 0.40 to 0.80 , with an rms scatter of about 0.17 . Although one could argue about the accuracy of the absolute values displayed in Figs. 14 and 16, there can however be no doubt that the distribution of spectral index over G78.2+2.1 is not uniform.

We note that the integrated spectral index quoted by Kothes et al. (2006) and calculated using the integrated CGPS flux densities is intermediate between our value and that of Zhang et al. (1997). However such a comparison, without being pointless, has nonetheless its limits since the two methods differ widely: the integrated flux density method is dominated by the regions of most intense emission, whereas the TT-plot technique is based on pixel statistics and will in fact neglect, so to speak, brighter regions if they represent only a small number of pixels.

It is now generally accepted that the relativistic electrons responsible for the synchrotron emission are produced by the Fermi acceleration mechanism (Bell 1978a,b; Blandford \& Ostriker 1978). This process predicts a spectral index given by

$\beta=2+\left(M^{2}+3\right) /\left[2\left(M^{2}-1\right)\right]$ 


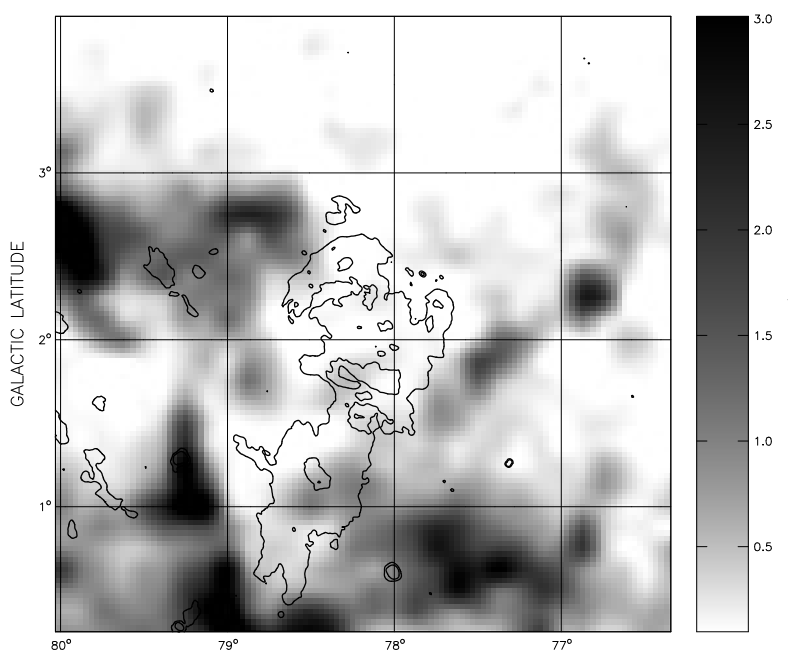

GALACTIC LONGITUDE

Fig. 24. Average of $\mathrm{CO}$ emission over $\sim 8 \mathrm{~km} \mathrm{~s}^{-1}$ centered at $6 \mathrm{~km} \mathrm{~s}^{-1}$.

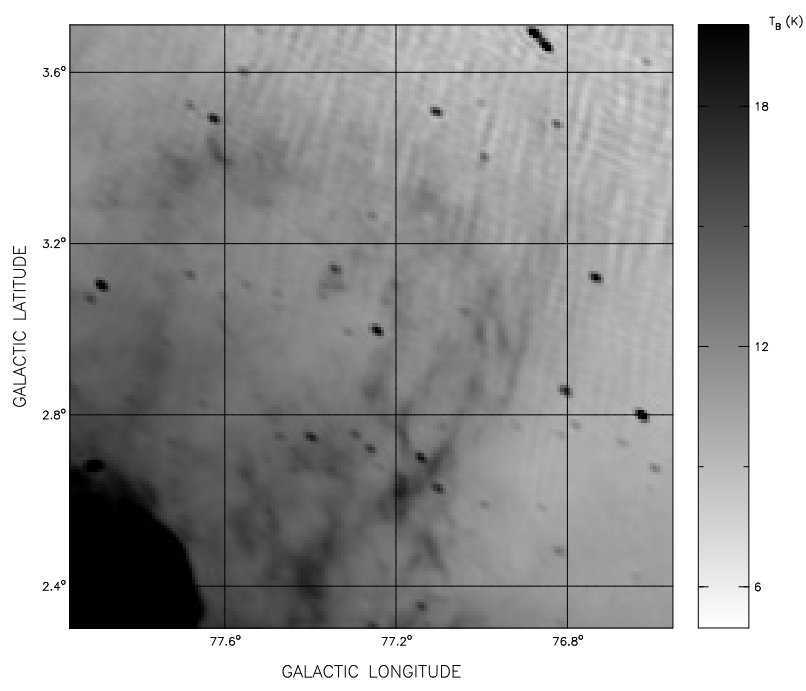

Fig. 25. Continuum image at $1420 \mathrm{MHz}$ showing the presence of a faint shell to the north-west.

where $M$ is the Mach number of the shock. For a very strong shock, $M$ tends towards 2.5 whereas a weaker shock produces a steeper spectrum. An average value of $\beta=2.75$ corresponds to $M=3$. The spottiness and large spatial variations of the spectral index distribution (e.g., Fig. 16) suggest that the shock wave is interacting with a highly inhomogeneous medium. An interpretation of the spectral index variations along similar lines has been successfully given for the SNR CTA1 (Pineault et al. 1997).

\subsection{Models based on HI kinematics}

On the basis of their earlier observations, Landecker et al. (1980) had proposed a model in which the SNR progenitor exploded in a thick slab of gas at a systemic velocity of $-5 \mathrm{~km} \mathrm{~s}^{-1}$. Noting the presence of a thick band of emission at about $20 \mathrm{~km} \mathrm{~s}^{-1}$ and a morphologically similar absorption band at about $-5 \mathrm{~km} \mathrm{~s}^{-1}$ (this band crosses the SNR from north to south-west and intersects the SNR continuum emission roughly where the emission is brightest), they proposed that an expanding shell of $\mathrm{HI}$ was present outside the shock front, with an expansion velocity of $25 \mathrm{~km} \mathrm{~s}^{-1}$. In this model (Fig. 36), the emission band at $20 \mathrm{~km} \mathrm{~s}^{-1}$ corresponds to the part of the shell on the far side of

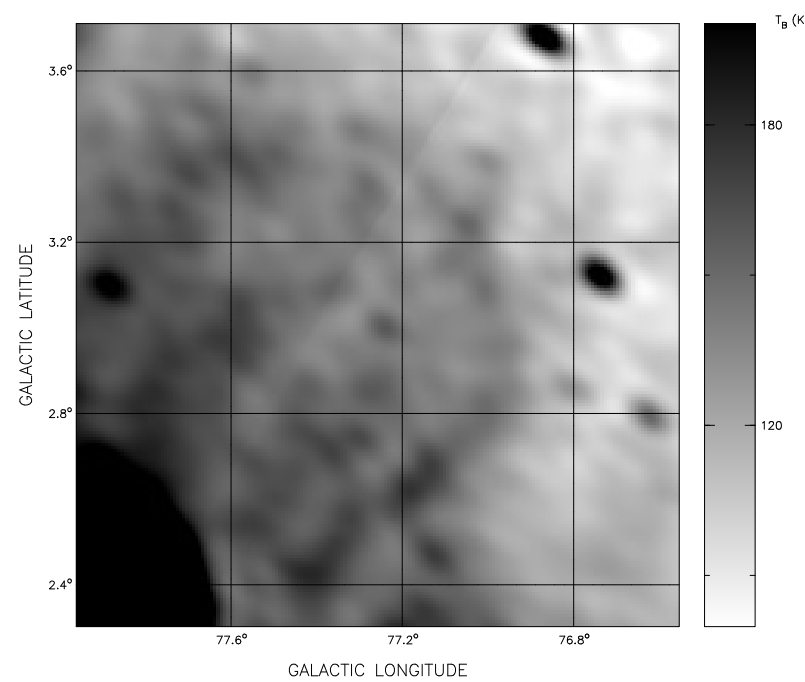

Fig. 26. Continuum image at $408 \mathrm{MHz}$ showing the presence of the same faint shell.

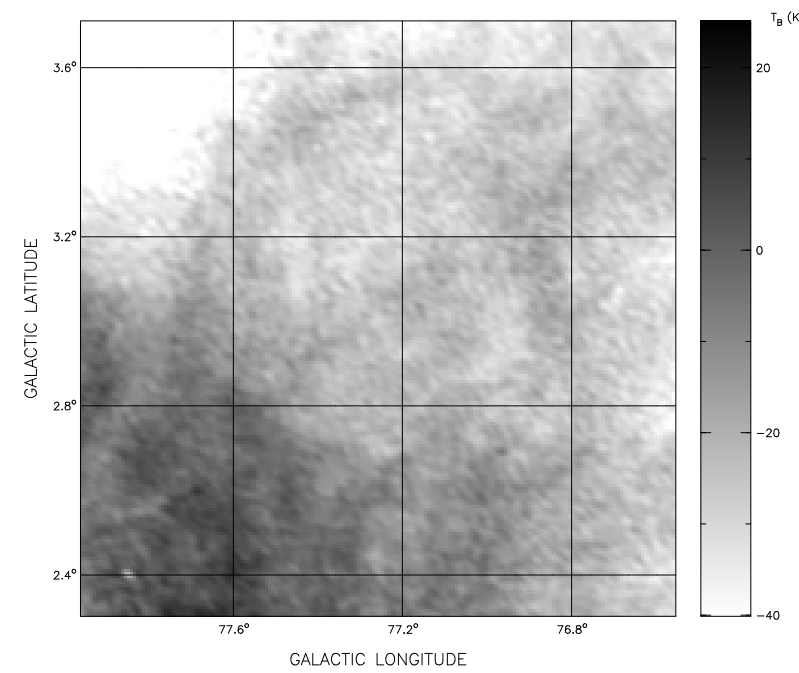

Fig. 27. Average of H I emission from -4 to $-12 \mathrm{~km} \mathrm{~s}^{-1}$.

the SNR, whereas the absorption band at $-5 \mathrm{~km} \mathrm{~s}^{-1}$ is on the near side inside the slab.

In the previous section, we have shown evidence for two partial H I shells or cavities (Figs. 21 and 23) which, taken together, outline the entire SNR. The first one extends from north through east to south-west and has a mean systemic velocity of $+5.5 \mathrm{~km} \mathrm{~s}^{-1}$, whereas the second one to the north-west is at $-14 \mathrm{~km} \mathrm{~s}^{-1}$. If we take the SNR systemic velocity as the mean of these two values, i.e. about $-5 \mathrm{~km} \mathrm{~s}^{-1}$, we can then envisage the SNR as interacting with two H I clouds as shown in Fig. 37. The emission band at $20 \mathrm{~km} \mathrm{~s}^{-1}$ is unrelated to the SNR and located behind it. Taking the systematic velocity as indicative of the distance and using Fig. 18 places the SNR at about $4 \mathrm{kpc}$ with a considerable error.

A variation on the preceding scenario is to suppose that the SNR systemic velocity corresponds to the most complete of the two partial shells, namely the one at $+5.5 \mathrm{~km} \mathrm{~s}^{-1}$. Figure 38 illustrates this case. The deep absorption band at $-5 \mathrm{~km} \mathrm{~s}^{-1}$ has to be in gas located in front of the SNR and moving away from it (towards us) with a relative velocity of about $10 \mathrm{~km} \mathrm{~s}^{-1}$. A systemic velocity of $+5.5 \mathrm{~km} \mathrm{~s}^{-1}$ corresponds to a distance of about $1 \mathrm{kpc}$, again with a considerable error. 


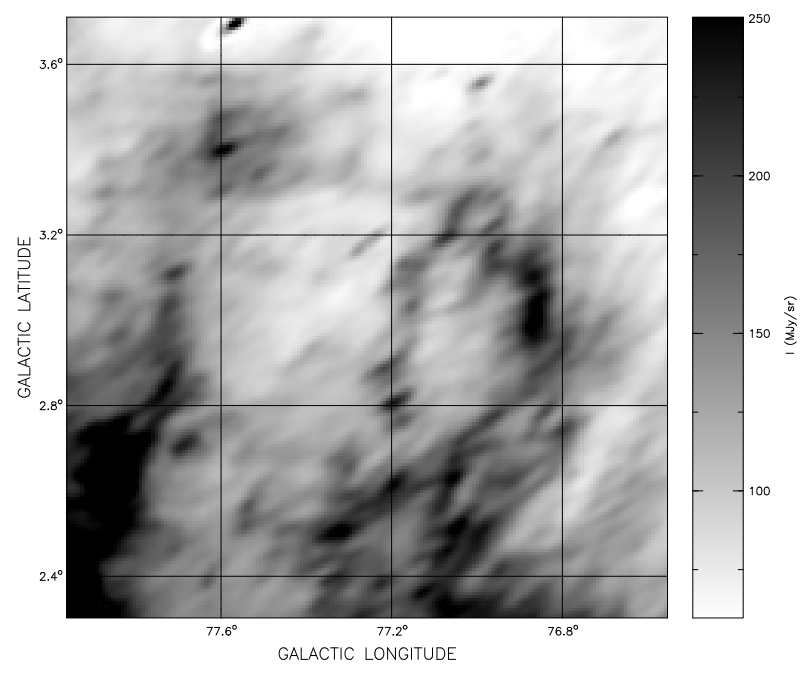

Fig. 28. $60 \mu \mathrm{m}$ image of the same field as the previous figure.

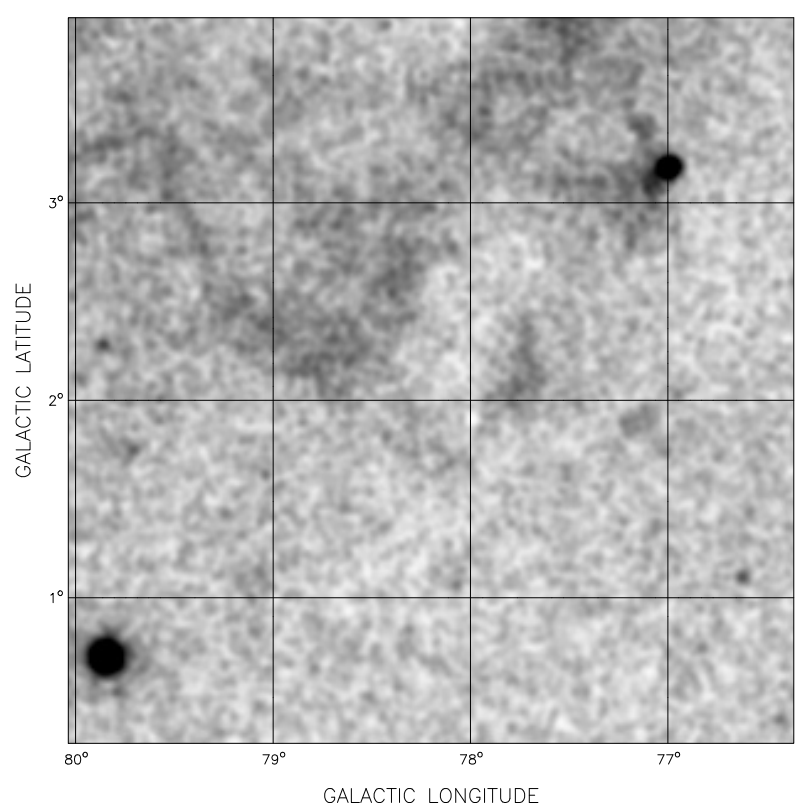

Fig. 29. ROSAT X-ray emission between 0.1 and $2.4 \mathrm{keV}$ in relative units.

Although both of the previous scenarios are possible, they are rather contrived. Our favoured scenario is one which takes into account the probable history of G78.2+2.1. The SNR progenitor is likely to have been through a strong stellar wind phase which would have largely evacuated a cavity around the star. We thus suppose that an expanding shell of H I has been set in motion by this wind. Putting the SNR at a systemic velocity of the first $\mathrm{H}$ I shell at $+5.5 \mathrm{~km} \mathrm{~s}^{-1}$ (also the velocity at which $\mathrm{CO}$ emission best matches the $\mathrm{HI}$ shell) and assuming the expanding wind-phase H I shell now has a velocity of $10 \mathrm{~km} \mathrm{~s}^{-1}$, we get the situation sketched in Fig. 39. The absorption in the approaching part of the progenitor's wind shell would be at an LSR velocity of about $-4 \mathrm{~km} \mathrm{~s}^{-1}$ and the receding far-side would be observed at a velocity of about $15 \mathrm{~km} \mathrm{~s}^{-1}$ thus explaining at least in part the emission band near $20 \mathrm{~km} \mathrm{~s}^{-1}$. Also, since the wide field $\mathrm{HI}$ CGPS images show that the emission band at $20 \mathrm{~km} \mathrm{~s}^{-1}$ is in fact part of a much longer thick H I filament extending well outside the outline of the SNR, its association with the SNR can be questioned. As in all scenarios, the second $-14 \mathrm{~km} \mathrm{~s}^{-1}$ partial shell

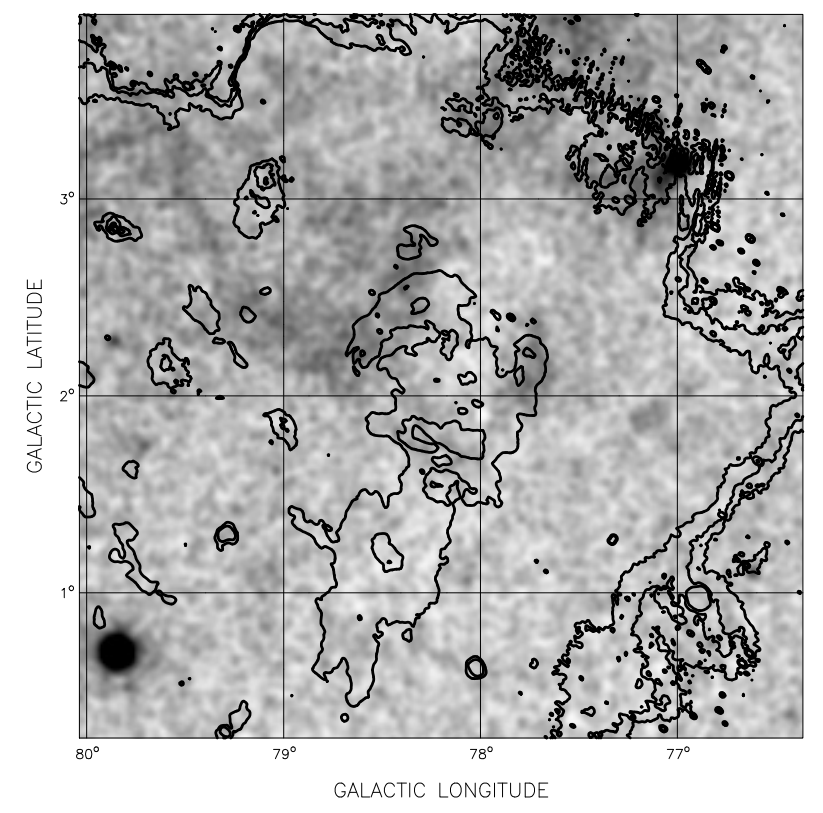

Fig. 30. ROSAT X-ray emission between 0.1 and $2.4 \mathrm{keV}$ in relative units, with $1420 \mathrm{MHz}$ continuum contours at 10.5, 11, 12, 28 and $50 \mathrm{~K}$.

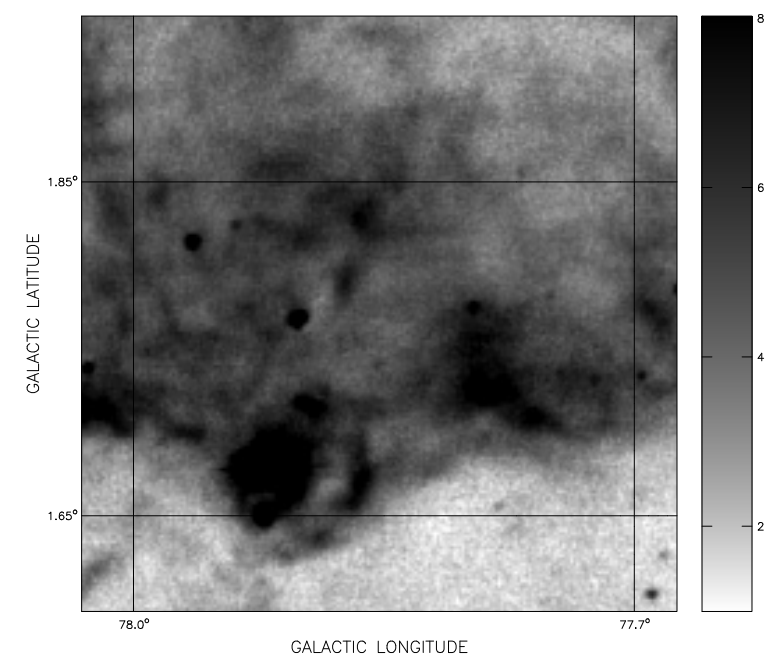

Fig. 31. MSX image at $8.23 \mu \mathrm{m}$ centered on the "bullet".

would have to originate in an $\mathrm{H}$ I structure having a significantly different LSR velocity, which is not a crucial constraint given the presence of complex motions in this region of the Galaxy (Lozinskaya 1992). In that case, there is no need to explain this feature in terms of the SNR kinematics. In any case, one should keep in mind that the existence of significant velocity dispersion and/or turbulence in the ISM makes assigning precise velocity values a somewhat perilous exercise, especially along this Galactic longitude. For this same reason, we hesitate to assign a firm distance to the SNR on the sole basis of H I kinematics.

\subsection{Other features of interest}

Two HI features with LSR velocities significantly different from the ones we have associated so far with the SNR are worth discussing further. They are the high-velocity cloudlet at $\sim 47 \mathrm{~km} \mathrm{~s}^{-1}$, and the weak $\mathrm{HI}$ arc at $\sim-68 \mathrm{~km} \mathrm{~s}^{-1}$. 


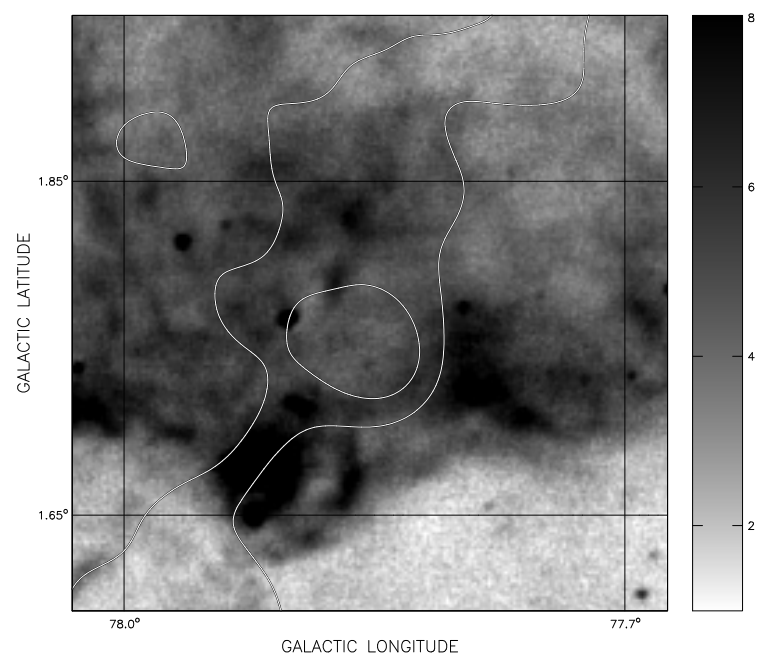

Fig. 32. Same as previous, but with $1420 \mathrm{MHz}$ radio continuum contours at 28 and $50 \mathrm{~K}$.
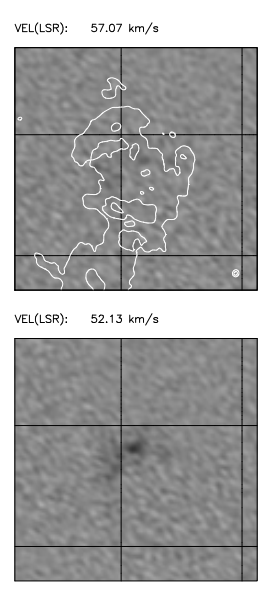

VELLLLP): $\quad 47.18 \mathrm{~km} / \mathrm{s}$

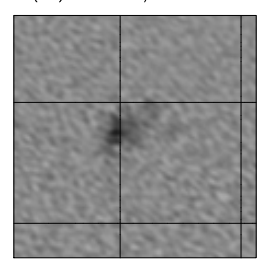

VELLLLSR): $42.23 \mathrm{~km} / \mathrm{s}$

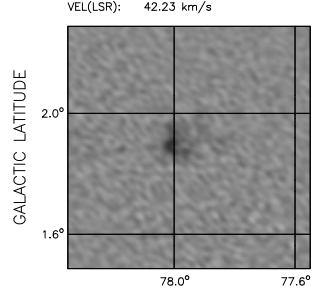

GALACTIC LONGITUDE

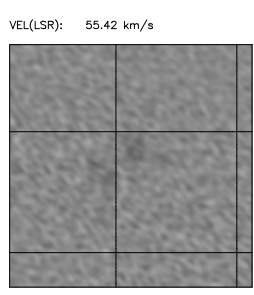

vEL(LSR): $\quad 50.48 \mathrm{~km} / \mathrm{s}$

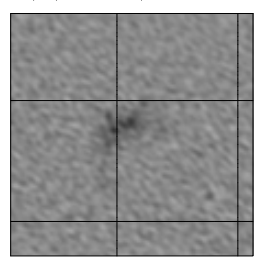

VELLLLS): $\quad 45.53 \mathrm{~km} / \mathrm{s}$

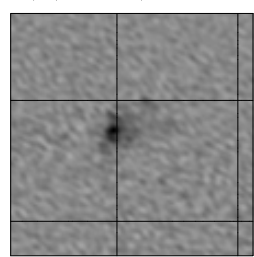

VELLLSRP): $\quad 40.58 \mathrm{~km} / \mathrm{s}$

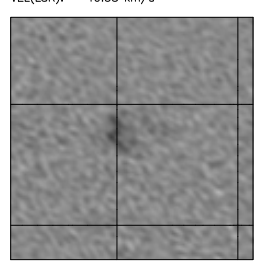

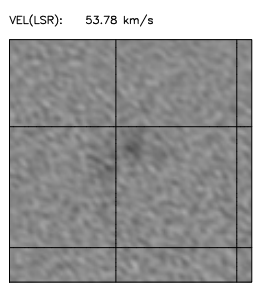

VELLLLFP): $43.83 \mathrm{~km} / \mathrm{s}$

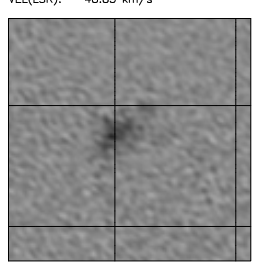

VELLLSR): $\quad 43 . .88 \mathrm{~km} / \mathrm{s}$

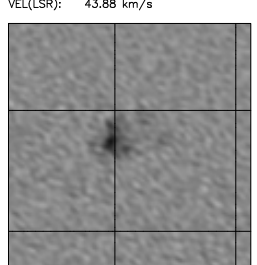

VELLLLSR): $38.94 \mathrm{~km} / \mathrm{s}$

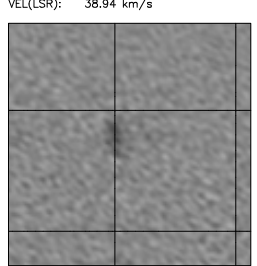

Fig. 33. A high-velocity cloudlet possibly accelerated by the SNR shock wave.

\subsubsection{High-velocity cloudlet}

This cloudlet appears projected at a distance from the SNR centre of about half the SNR radius. Hence, if we suppose it is moving radially away from the centre, then it is doing so at an inclination angle $i$ (with respect to the line of sight) of about $30^{\circ}(\sin i=0.5)$. It has thus been accelerated to a velocity $V_{\text {acc }}=\left|V_{\mathrm{o}}-V_{\text {sys }}\right| / \cos i$, where $V_{\mathrm{o}}=47 \mathrm{~km} \mathrm{~s}^{-1}$ and $V_{\text {sys }}$ are
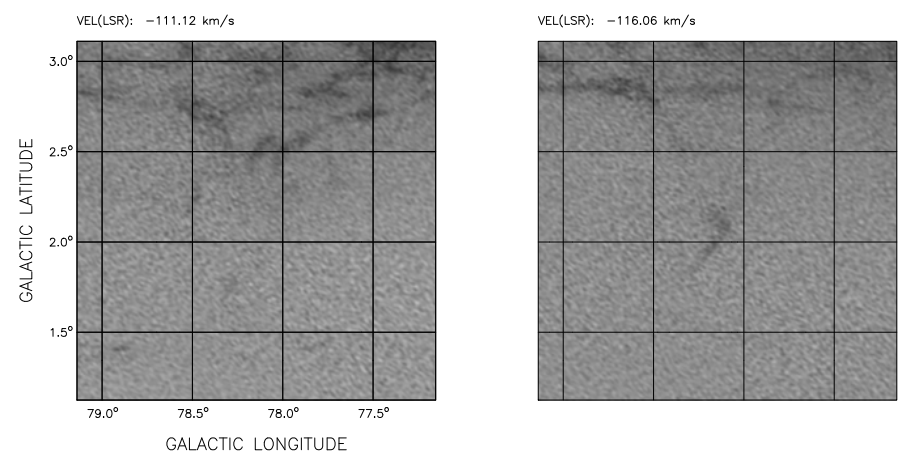

Fig. 34. H I emission showing the connection between a large-scale structure and a feature, in the image centre, previously believed to be a high-velocity cloudlet.

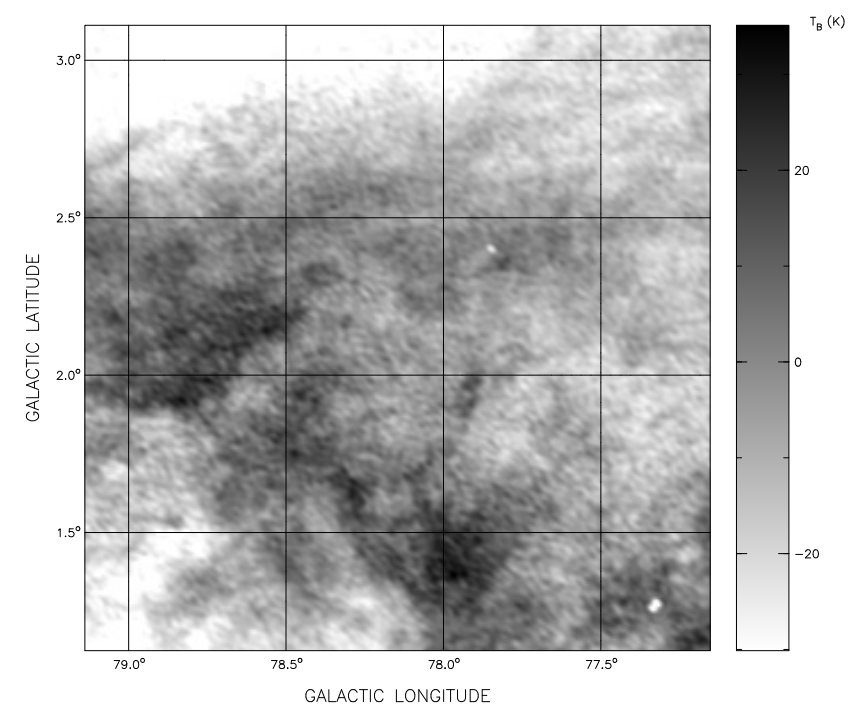

Fig. 35. Curving filament seen on an average of 5 channels between -67 and $-70 \mathrm{~km} \mathrm{~s}^{-1}$.

the observed LSR velocity and systemic velocity, respectively. The sign of the LSR velocity puts it on the far side of the SNR. The value of $V_{\text {acc }}$ is between 48 and $60 \mathrm{~km} \mathrm{~s}^{-1}$ depending on the value of the systemic velocity assumed $\left(+5.5\right.$ or $\left.-5 \mathrm{~km} \mathrm{~s}^{-1}\right)$. These velocities are not unreasonably large for any standard model of cloud acceleration, as pointed out by Landecker et al. (1980).

As mentioned before, the fact that this cloudlet is the only significantly emitting $\mathrm{H}$ I structure within a $6^{\circ} \times 6^{\circ}$ area centred on the SNR makes it highly probable that it is indeed an SNR-accelerated cloud. Its somewhat distorted appearance and velocity extent (Fig. 33) suggest that it may be in the process of evaporating into the ambient hotter gas inside the SNR cavity or breaking up as a result of its interaction with surrounding gas.

\subsubsection{Arc at $\sim-68 \mathrm{~km} \mathrm{~s}^{-1}$}

Unlike the cloudlet just discussed, this curving filament appears to be part of a more extended structure. If related to the SNR, it would be located on the approaching side with an inclination angle estimated at $\sim 30^{\circ}$ as well, thus implying an expansion velocity of between 73 and $85 \mathrm{~km} \mathrm{~s}^{-1}$ for $V_{\text {sys }}$ between -5 and $5.5 \mathrm{~km} \mathrm{~s}^{-1}$. This relatively high velocity makes it unlikely that this gas would have been set in motion during the wind phase 


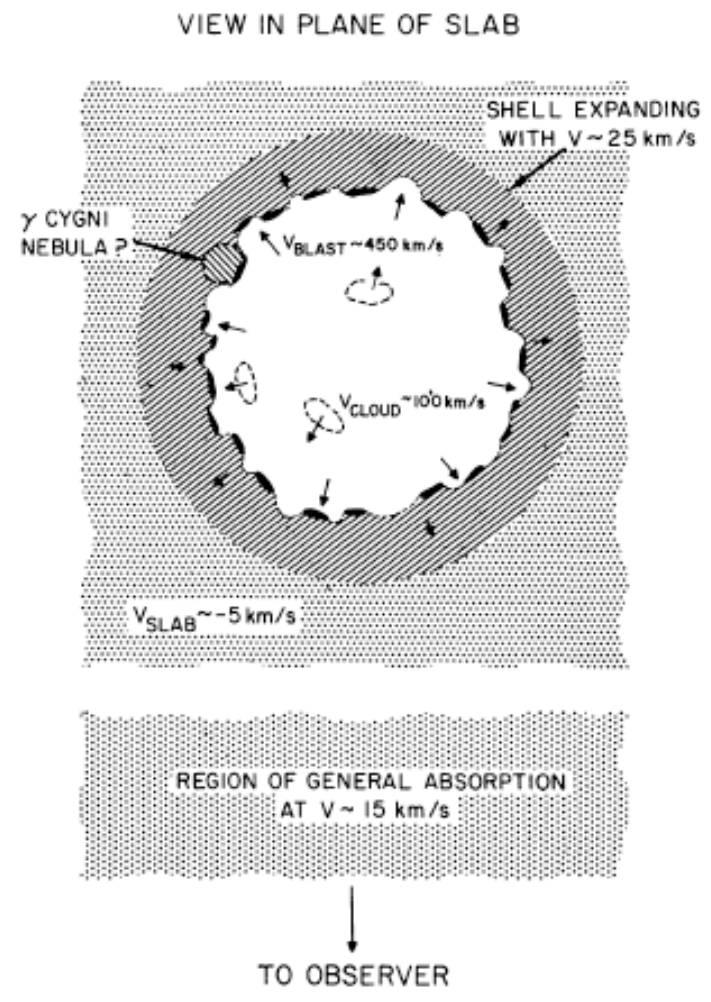

Fig. 36. A schematic view of the scenario envisaged by Landecker et al. (1980) to explain the H I dynamics. With the permission from Astronomy and Astrophysics.

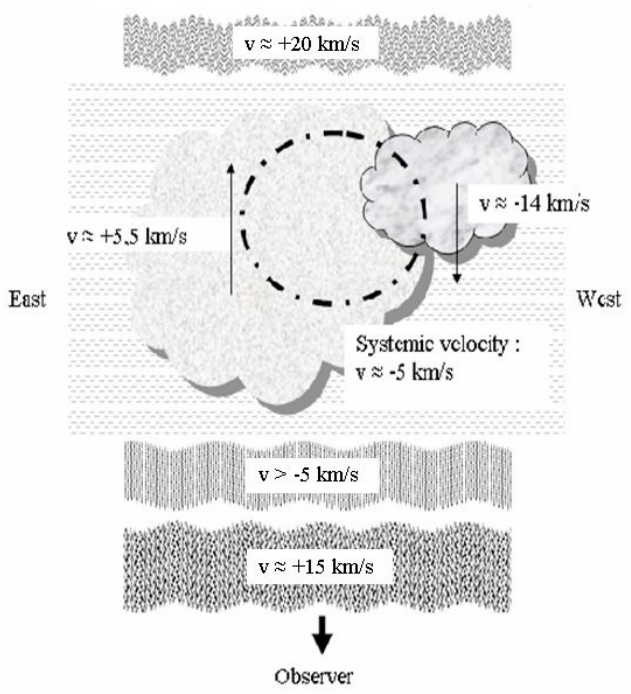

Fig. 37. A possible scenario based on the CGPS H I data (this paper).

of the SNR progenitor since the expansion velocity is given by (Lozinskaya 1992):

$v_{\exp }=16 n_{\mathrm{o}}^{-1 / 5} L_{36}^{1 / 5} t_{6}^{-2 / 5} \mathrm{~km} \mathrm{~s}^{-1}$,

where $n_{\mathrm{o}}$ is the unperturbed ISM number density in $\mathrm{cm}^{-3}, L_{36}=$ $L_{\mathrm{w}} /\left(10^{36} \mathrm{erg} \mathrm{s}^{-1}\right), L_{\mathrm{w}}$ is the wind power and $t_{6}=\left(t / 10^{6} \mathrm{yr}\right)$, $t$ being the duration of the wind phase.

Given the generally accepted young age of this SNR, it is also unlikely that gas shocked by the SNR blast wave would have had time to cool and form H I unless the unperturbed density of this structure was considerably higher than that of its

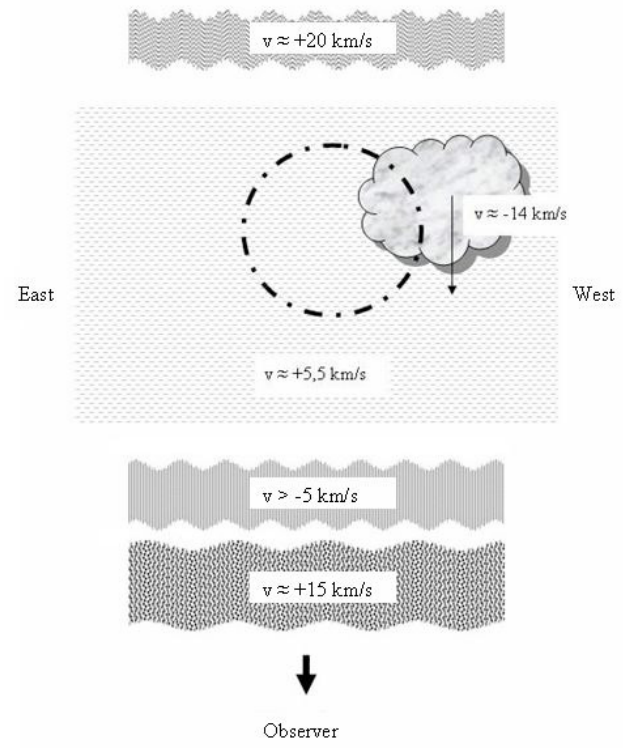

Fig. 38. Another plausible scenario.

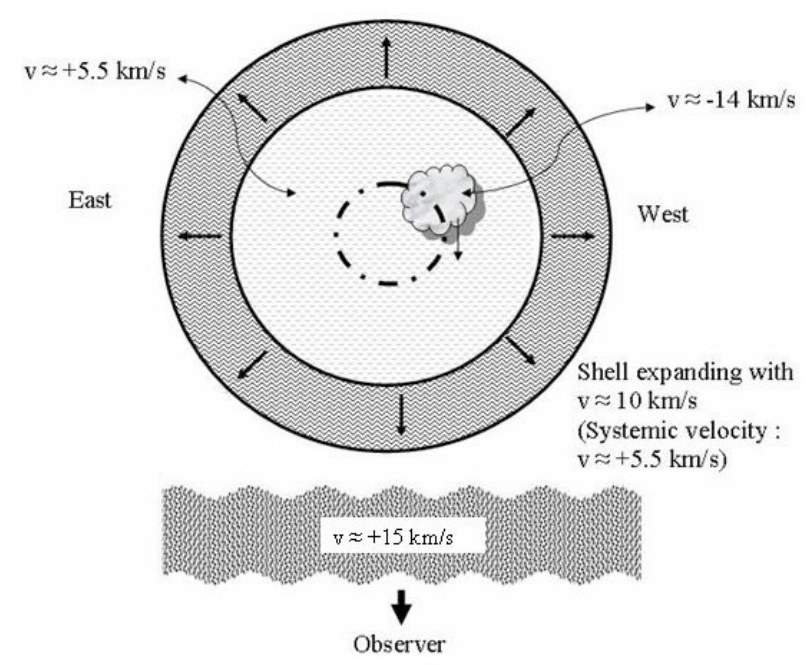

Fig. 39. Yet another plausible scenario.

surroundings. This suggests that this is an unrelated structure coincidentally seen along the same line of sight as the SNR.

\section{Conclusions}

1. After subtraction of the underlying thermal emission, we find that the radio continuum spectral index $\alpha$ between 408 and $1420 \mathrm{MHz}$, averaged over the entire SNR, is $0.75 \pm 0.03$. Without this subtraction, a lower value of $0.59 \pm 0.03$ is found, comparable within the errors to the integrated value estimated by Zhang et al. (1997). There are substantial spatial variations in spectral index, the lower values being found to the west and north-west and the higher ones to the south where continuum emission is strongest. The tomography technique, which allows one to keep the full resolution of the images, shows the spectral index varies from as low as 0.40 to as high as 0.80 with an rms dispersion of 0.17 .

2. The neutral hydrogen data show the presence of two complementary partial H I shells, at LSR velocities of 5.5 and $-14 \mathrm{~km} \mathrm{~s}^{-1}$ that appear to circumscribe the radio continuum 
emission. The first of these two structures coincides with a minimum or void of CO emission centered on the SNR.

3. A faint shell has been discovered to the north-west and is clearly visible on infrared and radio continuum images and also in the $\mathrm{H}$ I between -4 and $-12 \mathrm{~km} \mathrm{~s}^{-1}$. It could be the result of a blowout or breakout of the SNR blast wave into surrounding gas of lower density.

4. An H I cloudlet first discovered by Landecker et al. (1980) at $\sim 47 \mathrm{~km} \mathrm{~s}^{-1}$, and suggested to be accelerated by the SNR blast wave, shows considerable structure and is interpreted as being in the process of either dissolving within the hot SNR gas or breaking up as a result of interaction with neighbouring gas. Another suggested cloudlet near $-117 \mathrm{~km} \mathrm{~s}^{-1}$ turns out bo be part of a larger-scale structure and is not an SNR-accelerated cloudlet.

Acknowledgements. This work was supported by the Natural Sciences and Engineering Research Council of Canada (NSERC) and the Fonds FQRNT of Québec. The DRAO Synthesis Telescope is operated as a national facility by the National Research Council of Canada. The CGPS is a Canadian project with international partners and is supported by grants from NSERC. Data from the CGPS is publicly available through the facilities of the Canadian Astronomy Data Centre (http://cadc.hia.nrc.ca) operated by the Herzberg Institute of Astrophysics, NRC.

\section{References}

Anderson, M. C., \& Rudnick, L. 1993, ApJ, 408, 514

Anderson, M. C., Rudnick, L., Leppik, P., Perley, R., \& Braun, R. 1991, ApJ, 373,146

Bell, A. R. 1978a, MNRAS, 182, 147

Bell, A. R. 1978b, MNRAS, 182, 443

Blandford, R. D., \& Ostriker, J. P. 1978, ApJ, 221, L29

Brand, J., \& Blitz, L. 1993, A\&A, 295, 67

Braun, R., \& Strom, R. G. 1986, A\&AS, 63, 345

Broadbent, A., Haslam, C. G. T., \& Osborne, J. L. 1989, MNRAS, 237, 381

Cao, Y., Terebey, S., Prince, T. A., \& Beichman, C. A. 1997, ApJS, 111, 387

Cichowolski, S., Pineault, S., Arnal, E. M., et al. 2001, AJ, 122, 1938
Chastenay, P., \& Pineault, S. 1988, in Supernova Remnants and the Interstellar Medium, ed. R. S. Roger, \& T. L. Landecker (Cambridge: Cambridge University Press), IAU Coll., 101, 297

Egan, M. P., Price, S. D., Moshir, M. M., Cohen, M., \& Tedesco, E. 1999, The Midcourse Space Experiment Point Source Catalog Version 1.2 Explanatory Guide, Air Force Research Laboratory Technical Report, AFRL-VS-TR 1999-1522

Fowler, J. W., \& Aumann, H. H. 1994, in Science with High-Resolution FarInfrared Data, ed. S. Terebey, \& J. Mazzarella, JPL Publication, 94-5, 1

Fürst, E., Reich, W., \& Sofue Y. 1987, A\&AS, 71, 63

Gosachinskij, I. V. 2001, Astron. Lett., 27, 233

Higgs, L. A. 1977, AJ, 82, 329

Higgs, L. A., \& Tapping, K. F. 2000, AJ, 120, 2471

Higgs, L. A., Landecker, T. L., \& Roger, R. S. 1977, AJ, 82, 718

Katz-Stone, D. M., \& Rudnick, L. 1997, ApJ, 479, 258

Kothes, R., Fedotov, K., Foster, T. J., \& Uyaniker, B. 2006, A\&A, 457, 1081

Landecker, T. L., Roger, R., \& Higgs, L. A. 1980, A\&AS, 39, 133

Leung, H. O., \& Thaddeus, P. 1992, ApJS, 81, 267

Lozinskaya, T. A. 1992, Supernovae and Stellar Wind in the Interstellar Medium (New York: AIP)

Lozinskaya, T. A., Pravdikova, V. V., \& Finoguenov, A. V. 2000, Astron. Lett., 26, 77

Mavromatakis, F. 2003, A\&A, 408, 237

Moon, D. S., \& Koo, B. C. 1994, J. Kor. Astron. Soc., 27, 81

Pineault, S., \& Chastenay, P. 1990, MNRAS, 246, 169

Pineault, S., Landecker, T. L., Madore, B., \& Gaumont-Guay, S. 1993, AJ, 105, 1060

Pineault, S., Landecker, T. L., Swerdlyk, C. M., \& Reich, W. 1997, A\&A, 324, 1152

Pineault, S., Arnal, E. M., Cappa, C., et al. 2008, MNRAS, 386, 1739

Price, S. D., Egan, M. P., Carey, S., Mizuno, D., \& Kuchar, T. 2001, AJ, 121, 2819

Reich, W., Reich, P., \& Fürst, E. 1997, A\&AS, 126, 413

Roger, R. S., Costain, C. H., Lacey, J. D., Landecker, T. L., \& Bowers, F. K. 1973, Proc. IEE, 61, 1270

Taylor, A. R., Gibson, S. J., Peracaula, M., et al. 2003, AJ, 125, 3145 (CGPS)

Uchiyama, Y., Takahashi, T., Aharonian, F. A., \& Mattox, J. R. 2002, ApJ, 571, 866

Wendker, H. J. 1970, A\&A, 4, 378

Wendker, H. J., Higgs, L. A., \& Landecker, T. L. 1991, A\&A, 241, 551

Willis, A. G. 1999, A\&AS, 136, 603

Zhang, X., Zheng, Y., Landecker, T. L., \& Higgs, L. A. 1997, A\&A, 324, 641 